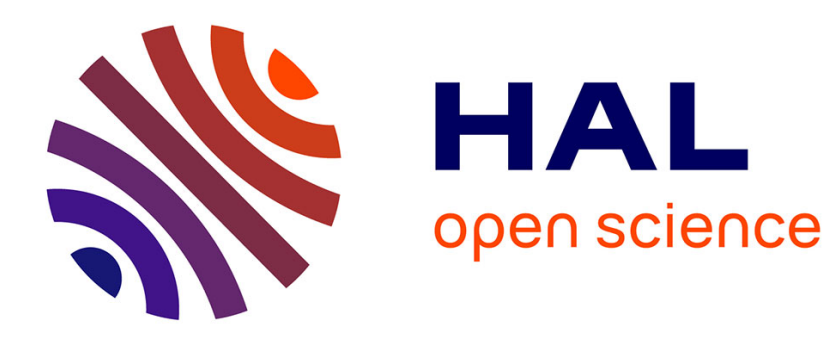

\title{
Nonnegative boundary control of 1D linear heat equations
}

Jérôme Lohéac

\section{To cite this version:}

Jérôme Lohéac. Nonnegative boundary control of 1D linear heat equations. Vietnam Journal of Mathematics, 2021, 49 (3), pp.845-870. 10.1007/s10013-021-00497-5 . hal-02613883v2

\section{HAL Id: hal-02613883 \\ https://hal.science/hal-02613883v2}

Submitted on 4 Jan 2021

HAL is a multi-disciplinary open access archive for the deposit and dissemination of scientific research documents, whether they are published or not. The documents may come from teaching and research institutions in France or abroad, or from public or private research centers.
L'archive ouverte pluridisciplinaire HAL, est destinée au dépôt et à la diffusion de documents scientifiques de niveau recherche, publiés ou non, émanant des établissements d'enseignement et de recherche français ou étrangers, des laboratoires publics ou privés. 


\title{
Nonnegative boundary control of 1D linear heat equations
}

\author{
Jérôme Lohéac*
}

January 4, 2021

\begin{abstract}
We consider the controllability of a one dimensional heat equation with nonnegative boundary controls. Despite the controllability in any positive time of this system, the unilateral nonnegativity control constraint causes a positive minimal controllability time. In this article, it is proved that at the minimal time, there exists a nonnegative control in the space of Radon measures, which consists of a countable sum of Dirac impulses.
\end{abstract}

Keywords: Minimal time, Nonnegative control, Dirac impulse, 1D heat equation.

\section{Contents}

\begin{tabular}{lll}
\hline & Introduction and main results & 1
\end{tabular}

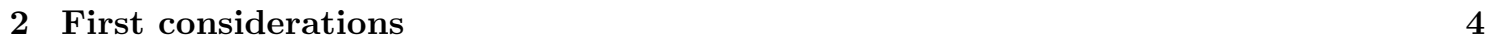

$\begin{array}{lll}3 & \text { Proof of Theorem } 1 & 6\end{array}$

\begin{tabular}{|lll}
\hline & Numerical example & 11
\end{tabular}

4.1 Dirichlet 1D heat . . . . . . . . . . . . . . . . . . . . . . . . . . . . . . . . . . . .

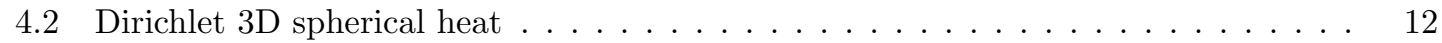

4.3 Coupled heat system . . . . . . . . . . . . . . . . . . . . 15

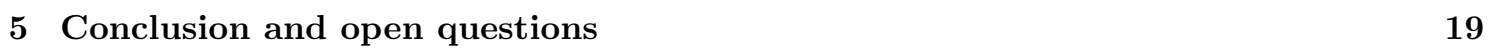

\begin{tabular}{|l|}
\hline A Controllability with nonnegative controls \\
\hline
\end{tabular}

B Existence of a nonnegative minimal time control in the space of Radon measures 24

\begin{tabular}{|l|l|l|l}
\hline C No gap situation & 25
\end{tabular}

\section{Introduction and main results}

In the recent years, controllability of partial differential equation with nonnegative control or with nonnegative state constraint has attracted many researchers [2, 4, 10, 11, 14, 15]. In the present paper, we are going to see, for one dimensional heat equation with nonnegative boundary control, that there exists a minimal controllability time, and that at this minimal time there exists a nonnegative control in the space of Radon measure which is the sum of a countable number of

*Université de Lorraine, CNRS, CRAN, F-54000 Nancy, France (jerome.loheac@univ-lorraine.fr). 
Dirac masses. Note that the existence of a minimal controllability time, and the existence of a nonnegative control, at the minimal controllability time, in the space of Radon measure were already proved in [11. Hence, the main novelty of this paper is the fact that this minimal time control can be taken as a countable sum of Dirac masses.

In order to precisely state the main result of the paper, we consider the one dimensional heat equation with boundary controls, whose state $y$ is given by

$$
\dot{y}(t, x)=\partial_{x}\left(p(x) \partial_{x} y(t, x)\right)+q(x) y(t, x) \quad(t>0, x \in(0,1)),
$$

with boundary conditions,

$$
\begin{aligned}
\alpha_{0} y(t, 0)+\alpha_{1} \partial_{x} y(t, 0) & =0 & & (t>0) \\
\beta_{0} y(t, 1)+\beta_{1} \partial_{x} y(t, 1) & =u(t) & & (t>0)
\end{aligned}
$$

and initial condition

$$
y(0, x)=y^{0}(x) \quad(x \in(0,1)) .
$$

We assume that $p \in C^{2}([0,1])$ is positive on $[0,1], q \in C([0,1]),\left|\alpha_{0}\right|+\left|\alpha_{1}\right|>0$ and $\left|\beta_{0}\right|+\left|\beta_{1}\right|>0$. Precise regularity condition on the initial state $y^{0}$ and on the control $u$ will be given later.

Let us also define the set of positive steady state

$$
\begin{aligned}
\mathcal{S}_{+}^{*}=\left\{\overline{\mathrm{y}} \in H^{2}(0,1) \mid \exists \overline{\mathrm{u}} \in \mathbb{R}_{+}^{*} \text { s.t. } \alpha_{0} \overline{\mathrm{y}}(0)+\alpha_{1} \partial_{x} \overline{\mathrm{y}}(0)=0, \quad \beta_{0} \overline{\mathrm{y}}(1)+\beta_{1} \partial_{x} \overline{\mathrm{y}}(1)=\overline{\mathrm{u}}\right. \\
\text { and } \left.\partial_{x}\left(p(x) \partial_{x} \overline{\mathrm{y}}(x)\right)+q(x) \overline{\mathrm{y}}(x)=0 \quad(\forall x \in(0,1))\right\} .
\end{aligned}
$$

Indeed, by linearity, it is easy to see that $\mathcal{S}_{+}^{*}$ is an open half line and is given by $\mathcal{S}_{+}^{*}=\mathbb{R}_{+}^{*} \overline{\mathrm{y}}^{1}$, with $\bar{y}^{1} \in H^{2}(0,1)$ solution of

$$
\partial_{x}\left(p(x) \partial_{x} \overline{\mathrm{y}}^{1}(x)\right)+q(x) \overline{\mathrm{y}}^{1}(x)=0 \quad(x \in(0,1)),
$$

with boundary conditions

$$
\alpha_{0} \overline{\mathrm{y}}^{1}(0)+\alpha_{1} \partial_{x} \overline{\mathrm{y}}^{1}(0)=0 \quad \text { and } \quad \beta_{0} \overline{\mathrm{y}}^{1}(1)+\beta_{1} \partial_{x} \overline{\mathrm{y}}^{1}(1)=1 .
$$

It has been shown in [7] that for every $y^{0} \in L^{2}(0,1)$, every time $T>0$ and every $m \in \mathbb{N}$, there exists a control $u \in C^{m}([0, T])$ such that the solution of $(1.1)$ satisfies $y(T, \cdot)=0$, and it is a trivial exercise to see that the same result holds for every target in $\mathcal{S}_{+}^{*}$. We also refer to [16] for some results on the controllability of the heat equation with steady state targets.

The controllability problem considered in this article is the following. Given some $y^{0} \in L^{2}(0,1)$ and some target $y^{1} \in \mathcal{S}_{+}^{*}$, find the minimum of the time $T>0$ such that there exists a nonnegative control $u \in L^{2}(0, T)$ steering the solution of $(1.1)$ from $y^{0}$ to $y^{1}$ in time $T$. This type of controllability problem has already been considered in [11. The results of 11] can be extended to the Proposition 1.1 below (see Appendix $\mathrm{A}$ for its proof). Before stating this result, let us define the operator $L \in \mathcal{L}\left(\mathcal{D}(L), L^{2}(0,1)\right)$ by

$$
\begin{aligned}
\mathcal{D}(L) & =\left\{\mathrm{y} \in H^{2}(0,1) \mid \alpha_{0} \mathrm{y}(0)+\alpha_{1} \partial_{x} \mathrm{y}(0)=0 \text { and } \beta_{0} \mathrm{y}(1)+\beta_{1} \partial_{x} \mathrm{y}(1)=0\right\}, \\
L \mathrm{y} & =\partial_{x}\left(p(x) \partial_{x} \mathrm{y}(x)\right)+q(x) \mathrm{y}(x) \quad(\mathrm{y} \in \mathcal{D}(L)) .
\end{aligned}
$$

Proposition 1.1. For every $y^{0} \in L^{2}(0,1)$ and every $y^{1} \in \mathcal{S}_{+}^{*}$, if one of the following condition is satisfied,

- $y^{0} \in \mathcal{S}_{+}^{*}$, or 
- $L$ is m-dissipative,

then there exist a time $T>0$ and a control $u \in L^{2}(0, T)$ steering the solution of the system (1.1) from $y^{0}$ to $y^{1}$ in time $T$;

We thus define,

$$
\begin{array}{r}
\underline{T}\left(y^{0}, y^{1}\right)=\inf \left\{T>0 \mid \exists u \in L^{2}(0, T) \text { s.t. } u \geqslant 0\right. \\
\text { and the solution } \left.y \text { of } 1.1 \text { satisfies } y(T, \cdot)=y^{1}\right\},
\end{array}
$$

note that if there does not exist such time $T>0$ (i.e., $y^{1}$ is not reachable from $y^{0}$ with nonnegative controls), we set $\underline{T}\left(y^{0}, y^{1}\right)=\infty$.

Finally, it can be shown, using similar arguments as the one used in [1] (see Appendix B), that in the minimal time $\underline{T}$, required to steer $y^{0}$ to $y^{1}$ with nonnegative controls, there exists a nonnegative control $\underline{u}$ in the space of Radon measures steering $y^{0}$ to $y^{1}$ in time $\underline{T}$. We thus introduce the space of Radon measure $\mathcal{M}([0, T])$, which are identified to Radon measures on $\mathbb{R}$ with support included in the compact set $[0, T] \subset \mathbb{R}$.

On the other hand, it has been shown in [12, that more precise results hold for finite dimensional control systems. More precisely, given $N \in \mathbb{N}^{*}, A \in \mathcal{M}_{N}(\mathbb{R})$ and $B \in \mathbb{R}^{N}$, we consider the (finite dimensional) control system

$$
\dot{Y}(t)=A Y(t)+B u(t) \quad(t>0)
$$

with initial condition

$$
Y(0)=Y^{0} \in \mathbb{R}^{N} .
$$

Similarly, we define the set of positive steady state,

$$
\Sigma_{+}^{*}=\left\{\bar{Y} \in \mathbb{R}^{N} \mid \exists \overline{\mathrm{u}} \in \mathbb{R}_{+}^{*} \text { s.t. } A \bar{Y}+B \overline{\mathrm{u}}=0\right\}
$$

and the minimal controllability time,

$$
\underline{\Theta}\left(Y^{0}, Y^{1}\right)=\left\{T>0 \mid \exists u \in L^{2}(0, T) \text { s.t. } u \geqslant 0\right.
$$

$$
\text { and the solution } Y \text { of } 1.5 \text { satisfies } Y(T)=Y^{1} \text {, }
$$

with $\underline{\Theta}\left(Y^{0}, Y^{1}\right)=\infty$ if $Y^{1}$ is not reachable from $Y^{0}$ in any time $T>0$. In [12, assuming that the pair $(A, B)$ is controllable, it has been shown that,

- if $Y^{0}, Y^{1} \in \Sigma_{+}^{*}$, then $\underline{\Theta}\left(Y^{0}, Y^{1}\right)<\infty$,

- if $\sigma(A) \subset \mathbb{R}_{-}^{*}+i \mathbb{R}$ and $Y^{1} \in \Sigma_{+}^{*}$, then $\underline{\Theta}\left(Y^{0}, Y^{1}\right)<\infty$ for every $Y^{0} \in \mathbb{R}^{N}$,

- if $\sigma(A) \subset \mathbb{R}$, and if $Y^{0}$ and $Y^{1} \in \mathbb{R}^{N}$ are such that $\underline{\Theta}\left(Y^{0}, Y^{1}\right)<\infty$, then there exist $t_{1}, \ldots, t_{\eta} \in$ $\left[0, \underline{\Theta}\left(Y^{0}, Y^{1}\right)\right]$ and $m_{1}, \ldots, m_{\eta} \in \mathbb{R}_{+}$such that the measure control $\underline{u}=\sum_{k=1}^{\eta} m_{k} \delta_{t_{k}}$ steers the solution of 1.5 from $Y^{0}$ to $Y^{1}$ in time $\underline{\Theta}\left(Y^{0}, Y^{1}\right)$, where $\eta \leqslant\lfloor(N+1) / 2\rfloor \in \mathbb{N}$.

The goal of this paper is to pass to the limit as $N \rightarrow \infty$ to obtain the following result for the infinite dimensional system (1.1). This strategy will lead to Theorem 1 below.

Theorem 1. Let $y^{0} \in L^{2}(0,1)$ and $y^{1} \in \mathcal{S}_{+}^{*}$ and assume that $y^{0} \neq y^{1}$ and $\underline{T}\left(y^{0}, y^{1}\right)<\infty$ (i.e., $y^{1}$ is reachable from $y^{0}$ with nonnegative controls). Then there exist an increasing sequence $\left(\tau_{i}\right)_{i \in \mathbb{N}^{*}} \in$ 
$\left[0, \underline{T}\left(y^{0}, y^{1}\right)\right)^{\mathbb{N}^{*}}$ and a sequence $\left(m_{i}\right)_{i \in \mathbb{N}^{*}} \in\left(\mathbb{R}_{+}^{*}\right)^{\mathbb{N}^{*}}$ such that the control $\underline{u} \in \mathcal{M}\left(\left[0, T\left(y^{0}, y^{1}\right)\right]\right)$ defined by

$$
\underline{u}(t)=\sum_{i=1}^{\infty} m_{i} \delta_{\tau_{i}}(t) \quad\left(t \in\left[0, \underline{T}\left(y^{0}, y^{1}\right)\right]\right)
$$

steers the solution of (1.1) from $y^{0}$ to $y^{1}$ in time $\underline{T}\left(y^{0}, y^{1}\right)$ (in $(1.8), \delta_{\tau}$ denotes the atomic mass located at time $\tau$ ).

Furthermore, we necessarily have $\lim _{i \rightarrow \infty} \tau_{i}=\underline{T}\left(y^{0}, y^{1}\right),\left(m_{i}\right)_{i \in \mathbb{N}^{*}} \in \ell^{1}$, and this control $\underline{u}$ is the unique nonnegative control, steering $y^{0}$ to $y^{1}$ in time $\underline{T}\left(y^{0}, y^{1}\right)$, in the set

$$
\mathcal{M}_{\delta}\left(\left[0, \underline{T}\left(y^{0}, y^{1}\right)\right]\right)=\left\{\sum_{i=1}^{\infty} \mu_{i} \delta_{\theta_{i}} \mid\left(\mu_{i}\right)_{i \in \mathbb{N}^{*}} \in \ell^{1},\left(\theta_{i}\right)_{i \in \mathbb{N}^{*}} \in\left[0, \underline{T}\left(y^{0}, y^{1}\right)\right]^{\mathbb{N}^{*}}\right\}
$$

of purely impulsive Radon measure.

Remark 1.2. Note that in the above result tells that the number od Dirac masses involved in the minimal time control is necessarily infinite, when $y^{1} \neq y^{0}$ and $y^{1} \in \mathcal{S}_{+}^{*}$. This will be a consequence of Lemma 3.3. However, when $y^{1}$ does not beolong to $\mathcal{S}_{+}^{*}$, it could happen that the minimal time control is composed of a finite number of Dirac masses.

Paper organization. We will first recall some well-known properties on Sturm-Liouville problems in Section 2. In this section, we will also recall the notion of solution for the problem 1.1 with Radon measure controls. The proof of Theorem 1 is contained in Section 3. In Section 4 numerical illustrations of this result are displayed. In particular, in Section 4.1. we consider (1.1) with $p=1, q=0$ and Dirichlet boundary control, in Section 4.2 , we consider the axisymmetric heat equation in the unit ball of $\mathbb{R}^{3}$ with Dirichlet boundary control, and finally, in Section 4.3 , we consider a coupled system of two 1D heat equations. Note that even if Theorem 1 does not apply to the systems considered in Sections 4.2 and 4.3 , we will see in these paragraphs that the results obtained can be adapted to these examples. Finally, Section 5 concludes this paper with some open questions and remarks. Note also that the results adapted from [11, 12] are given in Appendices A to C. In particular, in Appendix A, we prove Proposition 1.1, in Appendix B, we show that if $\underline{T}\left(y^{0}, y^{1}\right)<\infty$, then there exist a nonnegative Radon measure control steering $y^{0}$ to $y^{1}$ in time $\underline{T}\left(y^{0}, y^{1}\right)$, and in Appendix C, we show that the infimum time $\underline{T}\left(y^{0}, y^{1}\right)$ does not depend on the regularity ( $L^{2}$ or measure) of the control as soon as the target state belong to the set of positive steady states $\mathcal{S}_{+}^{*}$.

Notations. Dealing with classical sets, $\mathbb{N}$ is the set of nonnegative integers, $\mathbb{N}^{*}=\mathbb{N} \backslash\{0\}, \mathbb{R}$ is the set of real numbers, $\mathbb{R}_{+}$the set of nonnegative real numbers, and $\mathbb{R}_{+}^{*}=\mathbb{R}_{+} \backslash\{0\}$. For every $n \in \mathbb{N}^{*}, \mathcal{M}_{n}(\mathbb{R})$ is the set of $n \times n$ real matrices, and for $M \in \mathcal{M}_{n}(\mathbb{R})$, $\operatorname{ker} M$ denotes the null space of $M$. For every $s \in \mathbb{R},\lfloor s\rfloor$ is the integer part of $s$. We define $L^{2}(0,1)$ the set of square integrable real functions defined on $(0,1)$ and for every $T>0, L^{1}(0, T)$ is the set of integrable real functions defined on $(0, T)$. The set $\ell^{1}$ (respectively $\ell^{\infty}$ ) is the set of summable (respectively uniformly bounded) sequences $\left(c_{n}\right)_{n \in \mathbb{N}^{*}} \in \mathbb{R}^{\mathbb{N}^{*}}$. For every $k \in \mathbb{N}$ and every $T>0, C^{k}([0, T])$ denotes the set of $k$-differentiable real function defined on $[0, T]$. Finally, the time derivative is denoted with a dot and the space derivative with $\partial_{x}$.

\section{First considerations}

Some results on Sturm-Liouville problems. It is well-known (see e.g. [6] or [1, Theorem 2.29]) that the operator $L$, defined by [1.3), is self-adjoint and posses a sequence $\left(-\lambda_{n}\right)_{n \in \mathbb{N}^{*}} \in$ 
$\mathbb{R}^{\mathbb{N}^{*}}$ of distinct eigenvalues satisfying $\lambda_{1}<\cdots<\lambda_{n}<\lambda_{n+1}<\ldots$ with $\lambda_{n} \rightarrow \infty$ as $n \rightarrow \infty$. Furthermore, to each eigenvalue $-\lambda_{n}$ corresponds a single eigenfunction $\varphi_{n}$ of unitary norm, and the sequence of eigenfunction $\left\{\varphi_{n}\right\}_{n \in \mathbb{N}}$ forms an orthonormal basis of $L^{2}(0,1)$. Note also that, for every $n \in \mathbb{N}$, since $\varphi_{n}$ is a nontrivial solution of a second order ordinary differential equation, we necessarily have $\partial_{x} \varphi_{n}(1) \neq 0$, if $\beta_{0} \neq 0$, or $\varphi_{n}(1) \neq 0$, if $\beta_{1} \neq 0$.

Solution notion of 1.1 with Radon measure controls. The notion of solution of (1.1) with measure controls can be defined either by the transposition method or with the help of the spectral properties of $L$. Let us first recall that due to the Riesz Theorem, the set of Radon measure on $[0, T], \mathcal{M}([0, T])$ can be identified to the topological dual of continuous function on $[0, T]$. Furthermore, $\mathcal{M}([0, T])$ is a Banach space when endowed with the norm

$$
\|\underline{u}\|_{\mathcal{M}([0, T])}=\sup \left\{\int_{[0, T]} \varphi(t) \mathrm{d} \underline{u}(t) \mid \varphi \in C^{0}([0, T])\right\} \quad(\underline{u} \in \mathcal{M}([0, T])) .
$$

Definition by transposition. For this notion, we refer for instance to [5]. Given $y^{0} \in L^{2}(0,1)$ and $\underline{u} \in \mathcal{M}([0, T])$, we will say that $y$ is solution of $(1.1)$ in the sense of transposition if for every $\varphi \in \bar{C}^{2}([0, T] \times[0,1])$ satisfying

$$
\alpha_{0} \varphi(t, 0)+\alpha_{1} \partial_{x} \varphi(t, 0)=\beta_{0} \varphi(t, 1)+\beta_{1} \partial_{x} \varphi(t, 1)=0 \quad(t \in[0, T]),
$$

we have,

$$
\begin{aligned}
0=\int_{0}^{T} \int_{0}^{1}(-\dot{\varphi}( & \left.t, x)-\partial_{x}\left(p(x) \partial_{x} \varphi(t, x)\right)-q(x) \varphi(t, x)\right) y(t, x) \mathrm{d} x \mathrm{~d} t \\
& +\int_{0}^{1} y(T, x) \varphi(T, x) \mathrm{d} x-\int_{0}^{1} y^{0}(x) \varphi(0, x) \mathrm{d} x-\frac{p(1)}{\beta_{1}} \int_{0}^{T} \varphi(t, 1) \mathrm{d} \underline{u}(t),
\end{aligned}
$$

if $\beta_{1} \neq 0$, or

$$
\begin{aligned}
0=\int_{0}^{T} \int_{0}^{1}( & \left.-\dot{\varphi}(t, x)-\partial_{x}\left(p(x) \partial_{x} \varphi(t, x)\right)-q(x) \varphi(t, x)\right) y(t, x) \mathrm{d} x \mathrm{~d} t \\
& +\int_{0}^{1} y(T, x) \varphi(T, x) \mathrm{d} x-\int_{0}^{1} y^{0}(x) \varphi(0, x) \mathrm{d} x+\frac{p(1)}{\beta_{0}} \int_{0}^{T} \partial_{x} \varphi(t, 1) \mathrm{d} \underline{u}(t)
\end{aligned}
$$

if $\beta_{0} \neq 0$. This allows to define a week solution of 1.1 $y$ in $L^{\infty}\left(0, T ; H^{-s}(0,1)\right)$ for every $s>3 / 2$ (see e.g. [11, §2.2]) and the traces at times $t=0$ and $t=T$ has to be understood in the sense of 2.1).

Definition with spectral decomposition. Note also that taking $\varphi$ solution of $\dot{\varphi}=-L \varphi$ with $\varphi(T)=\varphi_{n}$, for $n \in \mathbb{N}^{*}$ and $\varphi_{n}$ the $n^{\text {th }}$ eigenfunction of $L$, we have $\varphi(t)=e^{-\lambda_{n}(T-t)} \varphi_{n}$ and injecting this relation in (2.1), we obtain,

$$
\int_{0}^{1} y(T, x) \varphi_{n}(x) \mathrm{d} x-e^{-\lambda_{n} T} \int_{0}^{1} y^{0}(x) \varphi_{n}(x) \mathrm{d} x=\gamma_{n} \int_{0}^{T} e^{-\lambda_{n}(T-t)} \mathrm{d} \underline{u}(t),
$$

with

$$
\gamma_{n}= \begin{cases}\frac{p(1)}{\beta_{1}} \varphi_{n}(1), & \text { if } \beta_{1} \neq 0, \\ -\frac{p(1)}{\beta_{0}} \partial_{x} \varphi_{n}(1), & \text { if } \beta_{0} \neq 0 .\end{cases}
$$


Since, as already recalled, the sequence $\left\{\varphi_{n}\right\}_{n \in \mathbb{N}^{*}}$ forms an orthogonal basis of $L^{2}(0,1)$, the relations (2.2) gives a definition of the trace of $y$ at time $t=T$ and also defines the controllability problem, i.e., given $y^{1} \in L^{2}(0,1)$, find $\underline{u} \in \mathcal{M}([0, T])$ such that 2.2 holds, for every $n \in \mathbb{N}^{*}$, with $y(T, \cdot)=y^{1}$.

Remark 2.1. Let us make some comments on the sequence $\left(\gamma_{n}\right)_{n}$. As already mentioned, we have $p(1)>0$ and $\varphi_{n}(1) \neq 0$ (respectively $\partial_{x} \varphi_{n}(1) \neq 0$ ) if $\beta_{1} \neq 0$ (respectively $\beta_{0} \neq 0$ ). This in particular ensures that $\gamma_{n} \neq 0$ for every $n \in \mathbb{N}^{*}$. Note also that we are dealing with an admissible boundary control operator (see e.g. 17] for this notion). Hence, if $\varphi_{n}$ is normalized so that $\left\|\varphi_{n}\right\|_{L^{2}(0,1)}=1$ for every $n \in \mathbb{N}^{*}$, we have $\sum_{n=1}^{\infty}\left|\gamma_{n}\right|^{2}=+\infty$ and $\sum_{n=1}^{\infty}\left|\frac{\gamma_{n}}{\lambda_{n}}\right|^{2}<\infty$.

In the rest of this paper, we will assume that $\left\|\varphi_{n}\right\|_{L^{2}(0,1)}=1$ for every $n \in \mathbb{N}^{*}$.

\section{Proof of Theorem 1}

Let us recall that we have assumed that $y^{1} \in \mathcal{S}_{+}^{*}$ is reachable from $y^{0}$ with nonnegative controls and that according to Appendices $\mathrm{B}$ and $\mathrm{C}$ we have,

$$
\begin{aligned}
\underline{T}\left(y^{0}, y^{1}\right)=\min & T \\
\mid \begin{array}{l} 
\\
\quad
\end{array} & \exists \underline{u} \in \mathcal{M}([0, T]) \text { s.t. } \underline{u} \geqslant 0 \text { and } y \text { solution of } 1.1] \text { satisfies } y(T)=y^{1} .
\end{aligned}
$$

Taking notion of solution with spectral decomposition (2.2), the minimization problem above becomes

$$
\begin{aligned}
& \min T \\
& \quad \begin{array}{l}
\quad T \geqslant 0, \\
\quad \underline{u} \in \mathcal{M}([0, T]) \text { s.t. } \underline{u} \geqslant 0 \text { and } \\
\quad Y_{n}^{1}-e^{-\lambda_{n} T} Y_{n}^{0}=\gamma_{n} \int_{0}^{T} e^{-\lambda_{n}(T-t)} \mathrm{d} \underline{u}(t), \quad\left(n \in \mathbb{N}^{*}\right),
\end{array}
\end{aligned}
$$

where, for every $n \in \mathbb{N}^{*}, \gamma_{n}$ is given by 2.3 , and we have set

$$
Y_{n}^{1}=\int_{0}^{1} y^{1}(x) \varphi_{n}(x) \mathrm{d} x \quad \text { and } \quad Y_{n}^{0}=\int_{0}^{1} y^{0}(x) \varphi_{n}(x) \mathrm{d} x .
$$

For every $N \in \mathbb{N}^{*}$, let us define,

$$
\begin{aligned}
& \underline{T}_{N}\left(y^{0}, y^{1}\right)=\min T \\
& \mid \begin{array}{l}
T \geqslant 0, \\
\exists \underline{u} \in \mathcal{M}([0, T]) \text { s.t. } \underline{u} \geqslant 0 \text { and } \\
Y_{n}^{1}-e^{-\lambda_{n} T} Y_{n}^{0}=\gamma_{n} \int_{0}^{T} e^{-\lambda_{n}(T-t)} \mathrm{d} \underline{u}(t), \quad(n \in\{1, \ldots, N\}) .
\end{array}
\end{aligned}
$$

Using the results contained in [12, it is easy to prove the following lemma.

Lemma 3.1. Let $y^{0} \in L^{2}(0,1)$ and $y^{1} \in \mathcal{S}_{+}^{*}$ and assume that $\underline{T}\left(y^{0}, y^{1}\right)<\infty$. For every $N \in \mathbb{N}^{*}$, we have $T_{N}\left(y^{0}, y^{1}\right) \leqslant T_{N+1}\left(y^{0}, y^{1}\right) \leqslant \underline{T}\left(y^{0}, y^{1}\right)$, and there exist $\eta \in\{1, \ldots,\lfloor(N+1) / 2\rfloor\}, \tilde{\tau}_{1}^{N}, \ldots, \tilde{\tau}_{\eta}^{N} \in$ $\left[0, T_{N}\left(y^{0}, y^{1}\right)\right.$ and $\tilde{m}_{1}^{N}, \ldots, \tilde{m}_{\eta}^{N} \in \mathbb{R}_{+}$such that the control $\underline{u}_{N} \in \mathcal{M}\left(\left[0, \underline{T}_{N}\left(y^{0}, y^{1}\right)\right]\right)$ defined by

$$
\underline{u}_{N}(t)=\sum_{i=1}^{\eta} \tilde{m}_{i}^{N} \delta_{\tilde{\tau}_{i}^{N}}(t) \quad\left(t \in\left[0, \underline{T}_{N}\left(y^{0}, y^{1}\right)\right]\right)
$$

is such that

$$
Y_{n}^{1}-e^{-\lambda_{n} \underline{T}_{N}\left(y^{0}, y^{1}\right)} Y_{n}^{0}=\gamma_{n} \int_{0}^{\underline{T}_{N}\left(y^{0}, y^{1}\right)} e^{-\lambda_{n}\left(\underline{T}_{n}\left(y^{0}, y^{1}\right)-t\right)} \mathrm{d} \underline{u}_{N} \quad(n \in\{1, \ldots, N\})
$$


holds. Furthermore, this control is the unique one in $\mathcal{M}\left(\left[0, \underline{T}_{N}\left(y^{0}, y^{1}\right)\right]\right)$ such that (3.4) holds.

In addition, there exists a constant $C\left(y^{0}, y^{1}\right)$ (only depending on $y^{0}$ and $\left.y^{1}\right)$ such that,

$$
\sum_{i=1}^{\eta} \tilde{m}_{i}^{N} \leqslant C\left(y^{0}, y^{1}\right) \quad\left(N \in \mathbb{N}^{*}\right)
$$

and there exists $\psi_{N}^{1}=\left(\left[\psi_{N}^{1}\right]_{1}, \ldots,\left[\psi_{N}^{1}\right]_{N}\right) \in \mathbb{R}^{N}$ such that

$$
\begin{array}{r}
\psi(t) \geqslant 0 \quad\left(t \in\left[0, \underline{T}_{N}\left(y^{0}, y^{1}\right)\right]\right) \text { and } \\
\left\{\tilde{\tau}_{i}^{N} \mid i \in\{1, \ldots, \eta\} \text { and } \tilde{m}_{i}^{N} \neq 0\right\} \subset\left\{t \in\left[0, \underline{T}_{N}\left(y^{0}, y^{1}\right)\right] \mid \psi(t)=0\right\},
\end{array}
$$

where $\psi(t)=\sum_{n=1}^{N} \gamma_{n} e^{-\lambda_{n}\left(\underline{T}_{n}-t\right)}\left[\psi_{N}^{1}\right]_{n}$

Proof. For every $N \in \mathbb{N}^{*}$, let us define,

$$
A_{N}=\left(\begin{array}{cccc}
-\lambda_{1} & 0 & \cdots & 0 \\
0 & \ddots & \ddots & \vdots \\
\vdots & \ddots & \ddots & 0 \\
0 & \cdots & 0 & -\lambda_{N}
\end{array}\right) \in \mathcal{M}_{N}(\mathbb{R}) \quad \text { and } \quad B_{N}=\left(\begin{array}{c}
\gamma_{1} \\
\vdots \\
\vdots \\
\gamma_{N}
\end{array}\right) \in \mathbb{R}^{N}
$$

It is then obvious that the minimization problem $(3.3)$ is exactly the minimization problem

$$
\begin{aligned}
& \underline{T}_{N}\left(y^{0}, y^{1}\right)=\min T \\
& \qquad \begin{aligned}
T \geqslant 0, \\
\exists \underline{u} \in \mathcal{M}([0, T]) \text { s.t. } \underline{u} \geqslant 0 \text { and the solution } Y \text { of } \dot{Y}=A_{N} Y+B_{N} \underline{u}, \\
\quad \text { with initial condition } Y(0)=\left(Y_{1}^{0}, \ldots, Y_{N}^{0}\right)^{\top}, \\
\quad \text { satisfies: } Y(T)=\left(Y_{1}^{1}, \ldots, Y_{N}^{1}\right)^{\top},
\end{aligned}
\end{aligned}
$$

where the reals $Y_{j}^{i}$ are defined in 3.2 .

Note that if $\underline{u} \in \mathcal{M}([0, T])$ is a nonnegative control steering the solution $y$ of $(1.1)$ from $y^{0}$ to $y^{1}$ in time $T>0$, then this control also steers the solution of $\dot{Y}=A_{N} Y+B_{N} \underline{u}$ from $Y^{0}$ to $Y^{1}$ in time $T$. Since $\underline{T}\left(y^{0}, y^{1}\right)<\infty$, such a time $T>0$ and a control exist, and this ensures, according to [12], that the minimization problem (3.3) admits a minimum. The fact that $T_{N}\left(y^{0}, y^{1}\right) \leqslant T_{N+1}\left(y^{0}, y^{1}\right) \leqslant$ $\underline{T}\left(y^{0}, y^{1}\right)$ is obvious, the upper bound on the sum of the $m_{i}^{N}$ can be obtained as in Appendix B (in particular, one can chose $\left.C\left(y^{0}, y^{1}\right)=e^{\left|\lambda_{0}\right| \underline{T}\left(y^{0}, y^{1}\right)}\left(e^{\left|\lambda_{0}\right| \underline{T}\left(y^{0}, y^{1}\right)}\left|\left\langle y^{0}, \varphi_{0}\right\rangle\right|+\left|\left\langle y^{1}, \varphi_{0}\right\rangle\right|\right) /\left|\gamma_{0}\right|\right)$, and the other claims of Lemma 3.1 directly follows from the results contained in [12, § 5.2]. by,

For every $N \in \mathbb{N}^{*}$, let us now define the sequences $m^{N}=\left(m_{i}^{N}\right)_{i \in \mathbb{N}^{*}} \in \ell^{1}$ and $\tau^{N}=\left(\tau_{i}^{N}\right)_{i \in \mathbb{N}^{*}} \in \ell^{\infty}$

$$
m_{i}^{N}=\left\{\begin{array}{ll}
\tilde{m}_{i}^{N} & \text { if } i \leqslant \eta, \\
0 & \text { otherwise }
\end{array} \quad \text { and } \quad \tau_{i}^{N}=\left\{\begin{array}{ll}
\tilde{\tau}_{i}^{N} & \text { if } i \leqslant \eta, \\
\underline{T}_{N}\left(y^{0}, y^{1}\right) & \text { otherwise }
\end{array} \quad\left(N \in \mathbb{N}^{*}, i \in \mathbb{N}^{*}\right),\right.\right.
$$

where $\eta=\eta(N), \tilde{m}_{i}^{N}$ and $\tilde{\tau}_{i}^{N}$ are defined in Lemma 3.1. It is obvious that we have,

$$
\left\|m^{N}\right\|_{\ell^{1}} \leqslant C\left(y^{0}, y^{1}\right) \quad \text { and } \quad\left\|\tau^{N}\right\|_{\ell^{\infty}} \leqslant \underline{T}\left(y^{0}, y^{1}\right) \quad\left(N \in \mathbb{N}^{*}\right),
$$

here also, $C\left(y^{0}, y^{1}\right)$ is defined by Lemma 3.1 . 
Lemma 3.2. There exist $m^{\infty} \in \ell^{1}$ and $\tau^{\infty} \in \ell^{\infty}$ satisfying $m_{i}^{\infty} \geqslant 0$ and $\tau_{i}^{\infty} \in\left[0, \underline{T}\left(y^{0}, y^{1}\right)\right]$, such that the control $\underline{u}_{\infty} \in \mathcal{M}\left(\left[0, \underline{T}\left(y^{0}, y^{1}\right)\right]\right)$ given by

$$
\underline{u}_{\infty}(t)=\sum_{i=1}^{\infty} m_{i}^{\infty} \delta_{\tau_{i}^{\infty}}(t) \quad\left(t \in\left[0, \underline{T}\left(y^{0}, y^{1}\right)\right]\right)
$$

is a control steering $y^{0}$ to $y^{1}$ in time $\underline{T}\left(y^{0}, y^{1}\right)$. Furthermore, the sequence of control $\left(\underline{u}_{N}\right)_{N \in \mathbb{N}^{*}}$ given by Lemma 3.1 is (up to the extraction of a subsequence) vaguely convergent to $\underline{u}_{\infty}$ in $\mathcal{M}\left(\left[0, \underline{T}\left(y^{0}, y^{1}\right)\right]\right)$.

Proof. In order to have more compact notations, we set $\underline{T}=\underline{T}\left(y^{0}, y^{1}\right), \underline{T}_{N}=\underline{T}_{N}\left(y^{0}, y^{1}\right)$ and, for $(i, n) \in\{0,1\} \times \mathbb{N}^{*}, Y_{n}^{i}$ is defined by 3.2 .

Part 1: The sequence $\left(\underline{T}_{N}\right)_{N \in \mathbb{N}^{*}}$ is convergent to $\underline{T}$, and the sequence $\left(\underline{u}_{N}\right)_{N \in \mathbb{N}^{*}}$ is vaguely convergent to some control $\underline{u}_{\infty} \in \mathcal{M}([0, \underline{T}])$.

According to Lemma 3.1. $\left(\underline{T}_{N}\right)_{N \in \mathbb{N}^{*}}$ is nondecreasing and bounded by $\underline{T}$. Hence, this sequence is convergent to some $\underline{T}_{\infty} \in[0, \underline{T}]$, and in fact, we have $\underline{T}_{\infty}=\underline{T}$. Indeed, let us define $u_{N}=$ $\sum_{i=1}^{\infty} m_{i}^{N} \delta_{\tau_{i}^{N}}$, this control steers the first $N$ moments $Y_{1}^{0}, \ldots, Y_{N}^{0}$ to $Y_{1}^{1}, \ldots, Y_{N}^{1}$ in time $\underline{T}_{N}$. This control is also bounded in $\mathcal{M}\left(\left[0, \underline{T}_{\infty}\right]\right)$ by some constant $C\left(y^{0}, y^{1}\right)$ independent of $N$ (see Lemma 3.1), and hence, up to a subsequence is vaguely convergent (see e.g. [3] for this notion and results) to some control $\underline{u}_{\infty} \in \mathcal{M}\left(\left[0, \underline{T}_{\infty}\right]\right)$ and we obtain that the control $\underline{u}_{\infty}$ steers all moments $\left(Y_{n}^{0}\right)_{n \in \mathbb{N}^{*}}$ to $\left(Y_{n}^{1}\right)_{n \in \mathbb{N}^{*}}$ in time $\underline{T}_{\infty}$. That is to say that $y^{0}$ is steered to $y^{1}$ in time $\underline{T}_{\infty} \leqslant \underline{T}$ with a nonnegative radon control. Since $\underline{T}$ is the minimal time, we necessarily have $\underline{T}_{\infty}=\underline{T}$.

Part 2: Properties on $\operatorname{supp} \underline{u}_{N}$.

Let us mention that for every $\varepsilon>0$, there exist $N \in \mathbb{N}^{*}$ such that $\operatorname{supp} \underline{u}_{N} \cap[\underline{T}-\varepsilon, \underline{T}] \neq \varnothing$. Indeed, assume by contradiction that for every $N \in \mathbb{N}^{*}, \operatorname{supp} \underline{u}_{N} \cap[\underline{T}-\varepsilon, \underline{T}]=\varnothing$. We then have, $\operatorname{supp} \underline{u}_{\infty} \subset[0, \underline{T}-\varepsilon]$, and $\underline{u}_{\infty}$ steers $y^{0}$ to $y^{1}$ in time $\underline{T}$. But, on one hand, $y^{1}$ is a steady state, we then have $y^{1} \in L^{2}(0,1) \backslash \mathcal{D}(L)$, and on the other hand, we have $y(\underline{T})=\sum_{n=1}^{\infty} e^{-\lambda_{n}} \underline{\underline{T}} Y_{n}^{0} \varphi_{n}+$ $\sum_{n=1}^{\infty} \gamma_{n} e^{-\lambda_{n} \varepsilon} \int_{[0, \underline{T}-\varepsilon]} e^{-\lambda_{n}(\underline{T}-\varepsilon-t)} \mathrm{d} \underline{u}_{\infty}(t) \varphi_{n} \in \mathcal{D}(L)$. This leads to a contradiction. As consequence, for every $K \in \mathbb{N}^{*}$ and every $\varepsilon>0$, there exist $N \in \mathbb{N}^{*}$ such that \# $\operatorname{supp} \underline{u}_{N} \cap[\underline{T}-\varepsilon, \underline{T}]>K$.

Part 3: Candidate for $\underline{u}_{\infty}$.

Let us define for every $i \in \mathbb{N}^{*}$ and every $N \in \mathbb{N}^{*}, M_{i}^{N}=\sum_{j=1}^{i} m_{j}^{N}$. Lemma 3.1 ensures that, for every $i \in \mathbb{N}^{*}$ and every $N \in \mathbb{N}^{*}$, we have $\tau_{i}^{N} \in[0, \underline{T}]$ and there exist a constant $C=C\left(y^{0}, y^{1}\right)$ such that $M_{i}^{N} \in[0, C]$. By compactness and diagonal extraction, there exist a subsequence of $\left(M^{N}, \tau^{N}\right)_{N \in \mathbb{N}^{*}}$ in $\ell^{\infty} \times \ell^{\infty}$ which is convergent to some $\left(M^{\infty}, \tau^{\infty}\right) \in \ell^{\infty} \times \ell^{\infty}$. In addition, we have for every $i \in \mathbb{N}^{*}, M_{i}^{\infty} \in[0, C]$ and $\tau_{i}^{\infty} \in[0, \underline{T}]$. Let us note that $0 \leqslant m_{i}^{N}=M_{i+1}^{N}-M_{i}^{N}$, ensuring that for every $i \in \mathbb{N}^{*}$, the sequence $\left(m_{i}^{N}\right)_{N \in \mathbb{N}^{*}}$ is convergent to $m_{i}^{\infty}=M_{i+1}^{\infty}-M_{i}^{\infty}$, and we have $m_{i}^{\infty} \geqslant 0$, and $\sum_{i=1}^{\infty} m_{i}^{\infty} \leqslant C$, that is to say that $m^{\infty} \in \ell^{1}$. Hence, the control defined by [3.6] is a natural candidate for steering $y^{0}$ to $y^{1}$ in time $\underline{T}$.

Part 4: Some property of $\left(m_{i}^{N}\right)$.

Let us now observe that for every $\varepsilon>0$, there exist $\tilde{N}$ such that $\left(\left|Y_{\tilde{N}}^{1}\right|+\left|Y_{\tilde{N}}^{0}\right|\right) /\left|\gamma_{\tilde{N}}\right| \leqslant \varepsilon$, and $\lambda_{\tilde{N}}>0$. The discussion made in Part 2, ensures the existence of $N_{0} \in \mathbb{N}^{*}$ such that $e^{-\lambda_{\tilde{N}}\left(\underline{T}-\tau_{N_{0}}^{\infty}\right)} \geqslant 1-\varepsilon$. Finally, for every $N \geqslant \tilde{N}$, we have

$$
\frac{Y_{\tilde{N}}^{1}-e^{-\lambda_{\tilde{N}} \underline{T}_{N}} Y_{\tilde{N}}^{0}}{\gamma_{\tilde{N}}}=\sum_{i=1}^{\infty} m_{i}^{N} e^{-\lambda_{\tilde{N}}\left(\underline{T}_{N}-\tau_{i}^{N}\right)}
$$

and hence,

$$
\sum_{i=N_{0}+1}^{\infty} m_{i}^{N} e^{-\lambda_{\tilde{N}}\left(\underline{T}_{N}-\tau_{i}^{N}\right)} \leqslant \varepsilon
$$


In addition, since $\left(\tau_{i}^{N}\right)_{i \in \mathbb{N}^{*}}$ is nondecreasing, we have,

$$
e^{-\lambda_{\tilde{N}}\left(\underline{T}-\tau_{N_{0}}^{N}\right)} \sum_{i=N_{0}+1}^{\infty} m_{i}^{N} \leqslant \varepsilon
$$

Finally, since $\left(\tau_{N_{0}}^{N}\right)_{N \in \mathbb{N}^{*}}$ goes to $\tau_{N_{0}}^{\infty}$ as $N \rightarrow \infty$, we deduce the existence of $N_{1} \in \mathbb{N}^{*}$ such that for every $N \geqslant N_{1}$, we have $e^{-\lambda_{\tilde{N}}\left(\underline{T}-\tau_{N_{0}}^{N}\right)} \geqslant(1-\varepsilon) / 2$. We have then obtained for $N \geqslant N_{1}$,

$$
\sum_{i=N_{0}+1}^{\infty} m_{i}^{N} \leqslant \frac{2 \varepsilon}{1-\varepsilon}
$$

In addition, since $m^{\infty} \in \ell^{1}$, the previous $N_{0}$ can be chosen large enough so that $\sum_{i=N_{0}+1}^{\infty} m_{i}^{\infty} \leqslant \varepsilon$.

Part 5: Vague convergence of $\underline{u}_{N}$ to $\underline{u}_{\infty}$.

Let us now prove that $\underline{u}_{N}=\sum_{i=1}^{\infty} m_{i}^{N} \delta_{\tau_{i}^{N}}$ is vaguely convergent to $\underline{u}_{\infty}=\sum_{i=1}^{\infty} m_{i}^{\infty} \delta_{\tau_{i}^{\infty}}$ in $\mathcal{M}([0, \underline{T}])$.

Note that here, we still denote by $\underline{u}_{N}$ the trivial extension of the original measure $\underline{u}_{N}$ on $[0, \underline{T}]$.

To prove the vague convergence, we consider $\varphi \in C^{0}([0, \underline{T}])$, such that $\|\varphi\|_{L^{\infty}(0, \underline{T})} \leqslant 1$, and $\varepsilon>0$. Then for every $N \in \mathbb{N}^{*}$ large enough (see Part 4),

$$
\begin{aligned}
&\left|\int_{[0, \underline{T}]} \varphi(t) \mathrm{d}\left(\underline{u}_{\infty}-\underline{u}_{N}\right)(t)\right| \leqslant \sum_{i=1}^{\infty}\left|m_{i}^{\infty} \varphi\left(\tau_{i}^{\infty}\right)-m_{i}^{N} \varphi\left(\tau_{i}^{N}\right)\right| \\
& \leqslant \sum_{i=1}^{N_{0}}\left|m_{i}^{\infty} \varphi\left(\tau_{i}^{\infty}\right)-m_{i}^{N} \varphi\left(\tau_{i}^{N}\right)\right|+\|\varphi\|_{L^{\infty}(0, T)}\left(\sum_{i=N_{0}+1}^{\infty} m_{i}^{\infty}+\sum_{i=N_{0}+1}^{\infty} m_{i}^{N}\right) \\
& \quad \leqslant \sum_{i=1}^{N_{0}}\left|m_{i}^{\infty} \varphi\left(\tau_{i}^{\infty}\right)-m_{i}^{N} \varphi\left(\tau_{i}^{N}\right)\right|+\varepsilon+\frac{2 \varepsilon}{1-\varepsilon},
\end{aligned}
$$

with $N_{0} \in \mathbb{N}^{*}$ defined in Part 4. By continuity of $\varphi$ and the convergence of $\left(m_{i}^{N}, \tau_{i}^{N}\right)_{N}$ to $\left(m_{i}^{\infty}, \tau_{i}^{\infty}\right)$ for $i \in\left\{1, \ldots, N_{0}\right\}$, we get the existence of $N_{1} \in \mathbb{N}^{*}$ such that for every $N \geqslant N_{1}$,

$$
\left|\int_{[0, \underline{T}]} \varphi(t) \mathrm{d}\left(\underline{u}_{\infty}-\underline{u}_{N}\right)(t)\right| \leqslant 2 \varepsilon+\frac{2 \varepsilon}{1-\varepsilon} .
$$

This ensures the vague convergence of $\underline{u}_{N}$ to $\underline{u}_{\infty}$ in $\mathcal{M}([0, \underline{T}])$, and this fact also ensures that $\underline{u}_{\infty}$ steers $y^{0}$ to $y^{1}$ in time $\underline{T}$.

In the sequences $\left(\tau_{i}^{\infty}\right)_{i}$ and $\left(m_{i}^{\infty}\right)_{i}$ given in the above lemma, it can happen that $\tau_{i}^{\infty}=\tau_{j}^{\infty}$ for some indexes $i \neq j$, or $m_{i}^{\infty}=0$ for some indexes $i$. But with a simple re-indexing, we have shown that there exist $I=I\left(y^{0}, y^{1}\right) \in \mathbb{N} \cup\{\infty\}$ and two sequences $\left(\tau_{i}\right)_{i=1, \ldots, I}$ and $\left(m_{i}\right)_{i=1, \ldots, I}$ such that, $\left(\tau_{i}\right)_{i}$ is a nondecreasing sequence in $\left[0, \underline{T}\left(y^{0}, y^{1}\right)\right], \sum_{i=0}^{I} m_{i}$ is finite, $m_{i}>0$ for every $i$, and

$$
Y_{n}^{1}-e^{-\lambda_{n} \underline{T}\left(y^{0}, y^{1}\right)} Y_{n}^{0}=\gamma_{n} \sum_{i=1}^{I} m_{i} e^{-\lambda_{n}\left(\underline{T}\left(y^{0}, y^{1}\right)-\tau_{i}\right)} \quad\left(n \in \mathbb{N}^{*}\right) .
$$

In the case that all masses $m_{i}$ are null, we set $I=0$ and by convention $\sum_{i=1}^{0} m_{i} e^{-\lambda_{n}\left(\underline{T}\left(y^{0}, y^{1}\right)-\tau_{i}\right)}=0$.

In the next lemma, we will show that we necessarily have $I=\infty$ and $\lim _{i \rightarrow \infty} \tau_{i}=\underline{T}\left(y^{0}, y^{1}\right)$ as soon as $y^{0} \neq y^{1}$. The proof of this lemma follows from the discussion made in the second part of the proof of Lemma 3.1

Lemma 3.3. Let $y^{0} \in L^{2}(0,1)$ and $y^{1} \in \mathcal{S}_{+}^{*}$ such that $y^{0} \neq y^{1}$ and $\underline{T}\left(y^{0}, y^{1}\right)<\infty$. Then,

1. $I>0$; 
2. there does not exist $I \in \mathbb{N}^{*}, 0 \leqslant \tau_{1}<\cdots<\tau_{I} \leqslant \underline{T}\left(y^{0}, y^{1}\right)$ and $m_{1}, \ldots, m_{I} \in \mathbb{R}_{+}^{*}$ such that the control $\underline{u}=\sum_{i=0}^{I} m_{i} \delta_{\tau_{i}}$ steers $y^{0}$ to $y^{1}$ in time $\underline{T}\left(y^{0}, y^{1}\right)$;

3. if there exist $0 \leqslant \tau_{1}<\cdots<\tau_{i}<\cdots \leqslant \underline{T}\left(y^{0}, y^{1}\right)$ and $\left(m_{i}\right)_{i \in \mathbb{N}^{*}} \in \ell^{1}$ such that $m_{i}>0$ for every $i \in \mathbb{N}$, and the control $\underline{u}=\sum_{i \in \mathbb{N}} m_{i} \delta_{\tau_{i}}$ steers $y^{0}$ to $y^{1}$ in time $\underline{T}\left(y^{0}, y^{1}\right)$, then we have $\lim _{i \rightarrow \infty} \tau_{i}=\underline{T}\left(y^{0}, y^{1}\right)$.

Proof. We set $\underline{T}=\underline{T}\left(y^{0}, y^{1}\right)$. Before entering the core of the proof, let us recall that according to Remark 2.1. we have assumed that the sequence $\left\{\varphi_{n}\right\}_{n \in \mathbb{N}^{*}}$ forms an orthonormal basis of $L^{2}(0,1)$, from which, we conclude that $\sum_{n=1}^{\infty}\left|\gamma_{n}\right|^{2}=+\infty$ and $\sum_{n=1}^{\infty} \lambda_{n}^{-2}\left|\gamma_{n}\right|^{2}<\infty$.

Proof of the $\mathbb{1}^{t}$ claim. If $I=0$, we have $Y_{n}^{1}=e^{-\lambda_{n} \underline{T}} Y_{n}^{0}$ for every $n \in \mathbb{N}^{*}$. Hence, if $\underline{T}=0$, we deduce that $y^{1}=y^{0}$ which is forbidden by assumption, and if $\underline{T} \neq 0$, we have $y^{1}=\sum_{n=1}^{\infty} Y_{n}^{1} \varphi_{n} \epsilon$ $L^{2}(0,1) \backslash \mathcal{D}(L)$ and $\sum_{n=1}^{\infty} e^{-\lambda_{n}} \underline{T} Y_{n}^{0} \varphi_{n} \in \mathcal{D}(L)$, leading to a contradiction.

Proof of the $2^{d d}$ claim. We consider the two possible situations, $\tau_{I}<\underline{T}$ and $\tau_{I}=\underline{T}$.

- If $\tau_{I}<\underline{T}$, then $\sum_{n=1}^{\infty}\left(e^{-\lambda_{n} \underline{T}} Y_{n}^{0}+\gamma_{n} \sum_{i=0}^{I} m_{i} e^{-\lambda_{n}\left(\underline{T}-\tau_{i}\right)}\right) \varphi_{n} \in \mathcal{D}(L)$, but $y^{1}=\sum_{n=1}^{\infty} Y_{n}^{1} \varphi_{n} \in$ $L^{2}(0,1) \backslash \mathcal{D}(L)$.

- If $\tau_{I}=\underline{T}$, then $\sum_{n=1}^{\infty}\left(Y_{n}^{1}-e^{-\lambda_{n}} \underline{T} Y_{n}^{0}-\gamma_{n} \sum_{i=0}^{I-1} m_{i} e^{-\lambda_{n}\left(\underline{T}-\tau_{i}\right)}\right) \varphi_{n} \in L^{2}(0,1)$, but $m_{I} \sum_{n=1}^{\infty} \gamma_{n} \varphi_{n} \notin$ $L^{2}(0,1)$.

Thus, in both cases, we have obtained a contradiction.

Proof of the $3^{d}$ claim. Since $\left(\tau_{i}\right)_{i \in \mathbb{N}^{*}}$ is an increasing and bounded sequence, there exists $\Theta \in(0, \underline{T}]$ such that $\Theta=\lim _{i \rightarrow \infty} \tau_{i}$. Let us assume by contradiction that $\Theta<\underline{T}$ and let $\tilde{T} \epsilon$ $(\Theta, \underline{T})$. We then have $\sum_{i=1}^{\infty} m_{i} e^{-\lambda_{n}\left(\underline{T}-\tau_{i}\right)}=e^{-\lambda_{n}(\underline{T}-\tilde{T})} \sum_{i=1}^{\infty} m_{i} e^{-\lambda_{n}\left(\tilde{T}-\tau_{i}\right)}$, from which, we deduce that $\sum_{n=1}^{\infty}\left(e^{-\lambda_{n} \underline{T}} Y_{n}^{0}+\gamma_{n} \sum_{i=1}^{\infty} m_{i} e^{-\lambda_{n}\left(\underline{T}-\tau_{i}\right)}\right) \varphi_{n} \in \mathcal{D}(L)$, which contradicts the fact that $y^{1} \in L^{2}(0,1) \backslash$ $\mathcal{D}(L)$.

Remark 3.4. Similarly, one can show that for every time $T>0$, every $y^{0} \in L^{2}(0,1)$ and every steady state $y^{1}\left(y^{1}\right.$ is not assumed to be a positive steady state) such that $y^{1} \neq y^{0}$ and $y^{1} \neq 0$, if the control $\underline{u} \in \mathcal{M}\left([0, T]\right.$ ) (here also we do not assume that $\underline{u}$ is nonnegative) steers $y^{0}$ to $y^{1}$ in time $T$, then $\underline{u}$ is not a finite sum of Dirac impulses.

Remark 3.5. Let us also mention that Lemma 3.3 also ensures that $\underline{T}_{N}\left(y^{0}, y^{1}\right)<\underline{T}\left(y^{0}, y^{1}\right)$ for every $N \in \mathbb{N}^{*}$. Indeed, assume by contradiction that there exist $N \in \mathbb{N}^{*}$ such that $\underline{T}_{N}\left(y^{0}, y^{1}\right)=$ $\underline{T}\left(y^{0}, y^{1}\right)$. Hence, the control $\underline{u}=\sum_{n=0}^{\infty} m_{i} \delta_{\tau_{i}}$, composed of an infinite Dirac masses, and the control $\underline{u}_{N}=\sum_{n=1}^{\infty} m_{i}^{N} \delta_{\tau_{i}^{N}}$, composed of at most $\lfloor(N+1) / 2\rfloor$ Dirac masses, steer the first $N$ moments of $y^{0}$ to the first $N$ moments of $y^{1}$ in time $\underline{T}_{N}\left(y^{0}, y^{1}\right)$. This leads to a contradiction with the uniqueness result stated in Lemma 3.1

In order to complete the proof of Theorem 1 it remains to prove the uniqueness of this control in the space of purely impulsive Radon measures. To this end, we will use the following lemma.

Lemma 3.6. Let $T>0,\left(\theta_{k}\right)_{k \in \mathbb{N}^{*}} \in([0, T])^{\mathbb{N}^{*}}$ and assume that $\theta_{k} \neq \theta_{j}$ for every $j \neq k$. Then, the family $\left\{\left(\gamma_{n} e^{-\lambda_{n}\left(T-\theta_{k}\right)}\right)_{n \in \mathbb{N}^{*}}\right\}_{k \in \mathbb{N}^{*}}$ is free in $\mathbb{R}^{\mathbb{N}^{*}}$.

Proof. Assume by contradiction that there exist $N \in \mathbb{N}^{*}$ and $\alpha^{1}, \ldots, \alpha^{N} \in \mathbb{R}$ not all null such that $\sum_{k=1}^{N} \alpha^{k} \gamma_{n} e^{-\lambda_{n}\left(T-\theta_{k}\right)}=0$ for every $n \in \mathbb{N}^{*}$.

This in particular implies that the matrix

$$
M=\left(\begin{array}{ccc}
\gamma_{1} e^{-\lambda_{1}\left(T-\theta_{1}\right)} & \cdots & \gamma_{1} e^{-\lambda_{1}\left(T-\theta_{N}\right)} \\
\vdots & & \vdots \\
\gamma_{N} e^{-\lambda_{N}\left(T-\theta_{1}\right)} & \cdots & \gamma_{N} e^{-\lambda_{N}\left(T-\theta_{N}\right)}
\end{array}\right) \in \mathcal{M}_{N}(\mathbb{R})
$$


is not invertible. This also implies the existence of $\psi^{1}=\left(\psi_{1}^{1}, \ldots, \psi_{N}^{1}\right)^{\top} \in \mathbb{R}^{N} \backslash\{0\}$ such that $\psi^{1} \in \operatorname{ker} M^{\top}$, that is to say,

$$
\sum_{n=1}^{N} \psi_{n}^{1} \gamma_{n} e^{-\lambda_{n}\left(T-\theta_{k}\right)}=0 \quad(k \in\{1, \ldots, N\}),
$$

Defining the map $\psi: t \in[0, T] \mapsto \sum_{n=1}^{N} \psi_{n}^{1} \gamma_{n} e^{-\lambda_{n}(T-t)} \in \mathbb{R}$, we deduce that $\psi$ admits $N$ distinct roots. According to [9, Exercice 13 p. 154], the function $\psi$ is either null or admits at most $N-1$ roots (counted with their multiplicity), we deduce that $\psi \equiv 0$, and finally with the controllability of the pair $\left(A_{N}, B_{N}\right)$ (defined by (3.5)), we deduce that we necessarily have $\psi^{1}=0$. This leads to a contradiction with the fact that $\psi^{\perp} \neq 0$.

We are now in position to prove the uniqueness of this control in the space of purely impulsive Radon measures. Indeed, if there exist two sequences, $\left(\tilde{m}_{i}, \tilde{\tau}_{i}\right)_{i \in \mathbb{N}^{*}},\left(m_{i}, \tau_{i}\right)_{i \in \mathbb{N}^{*}} \in\left(\mathbb{R}_{+}^{*} \times\right.$ $\left.\left[0, \underline{T}\left(y^{0}, y^{1}\right)\right)\right)^{\mathbb{N}^{*}}$ such that the controls $\underline{\tilde{u}}=\sum_{i=1}^{\infty} \tilde{m}_{i} \delta_{\tilde{\tau}_{i}}$ and $\underline{u}=\sum_{i=1}^{\infty} m_{i} \delta_{\tau_{i}}$ steer $y^{0}$ to $y^{1}$ in time $\underline{T}\left(y^{0}, y^{1}\right)$, we have,

$$
\gamma_{n} \sum_{i=1}^{\infty} \mu_{i} e^{-\lambda_{n}\left(T-\theta_{i}\right)}=0 \quad\left(n \in \mathbb{N}^{*}\right),
$$

where $\theta_{i} \neq \theta_{j}$ for $i \neq j,\left\{\theta_{i}, i \in \mathbb{N}^{*}\right\}=\left\{\tau_{i}, i \in \mathbb{N}^{*}\right\} \cup\left\{\tilde{\tau}_{i}, i \in \mathbb{N}^{*}\right\}$, and $\mu_{i}=\sum_{\substack{j \in \mathbb{N}^{*} \\ \tau_{j}=\theta_{i}}} m_{j}-\sum_{\substack{j \in \mathbb{N}^{*} \\ \tilde{\tau}_{j}=\theta_{i}}} \tilde{m}_{j}$. Thus, by application of Lemma 3.6. we deduce that we necessarily have $\mu_{i}=0$ for every $i$, that is to say that, $\underline{u}=\underline{\tilde{u}}$.

\section{Numerical example}

\subsection{Dirichlet 1D heat}

We consider the system (1.1) with $p \equiv 1, \alpha_{0}=\beta_{0}=1$ and $\alpha_{1}=\beta_{1}=0$. For this system, the set of positive steady states is given by $\mathcal{S}_{+}^{*}=\left\{x \in[0,1] \mapsto x v \mid v \in \mathbb{R}_{+}^{*}\right\}$. It is also classical that the corresponding eigenvalues and normalized eigenvectors of the operator $L$ defined by (1.3) are

$$
-\lambda_{n}=-(n \pi)^{2} \quad \text { and } \quad \varphi_{n}(x)=\sqrt{2} \sin (n \pi x) \quad(x \in[0,1]) \quad\left(n \in \mathbb{N}^{*}\right) .
$$

We also have

$$
\gamma_{n}=-\partial_{x} \varphi_{n}(1)=(-1)^{n+1} \sqrt{2} n \pi \quad\left(n \in \mathbb{N}^{*}\right) .
$$

This in particular implies that for every $y^{0} \in L^{2}(0,1)$ and every $y^{1} \in \mathcal{S}_{+}^{*}$, we have $\underline{T}\left(y^{0}, y^{1}\right)<\infty$ (see Proposition 1.1) and the result of Theorem 1 applies. Furthermore, as observed in Section 3 the minimal time control $\underline{u}$ given by Theorem 1 can be approximated by the sequence of minimizers of $(3.3)$.

For the numerical simulation, we consider $y^{1}(x)=x \in \mathcal{S}_{+}^{*}$ and $y^{0}(x)=\cos (\pi x) \in L^{2}(0,1)$, and we have

$$
\begin{aligned}
& Y_{n}^{0}=\int_{0}^{1} y^{0}(x) \varphi_{n}(x) \mathrm{d} x=\frac{\left(1+(-1)^{n}\right) \sqrt{2} n}{\pi\left(n^{2}-1\right)} \text { and } \\
& Y_{n}^{1}=\int_{0}^{1} y^{1}(x) \varphi_{n}(x) \mathrm{d} x=\frac{(-1)^{n+1} \sqrt{2}}{n \pi} . \quad\left(n \in \mathbb{N}^{*}\right) .
\end{aligned}
$$


In order to numerically solve (3.3), we use the sequential quadratic programming method of the optimization toolbox of matlab. On Figure 1, we display the values of $\underline{T}_{N}=\underline{T}_{N}\left(y^{0}, y^{1}\right), \tau_{i}^{N}$ and $m_{i}^{N}$ with respect to $N$. Recall that for some given $N \in \mathbb{N}^{*}$, the optimal control of (3.3) is a some of at most $\lfloor(N+1) / 2\rfloor$ Dirac masses and for $i>\lfloor(N+1) / 2\rfloor$, we have set $\tau_{i}^{N}=\underline{T}_{N}$ and $m_{i}^{N}=0$.
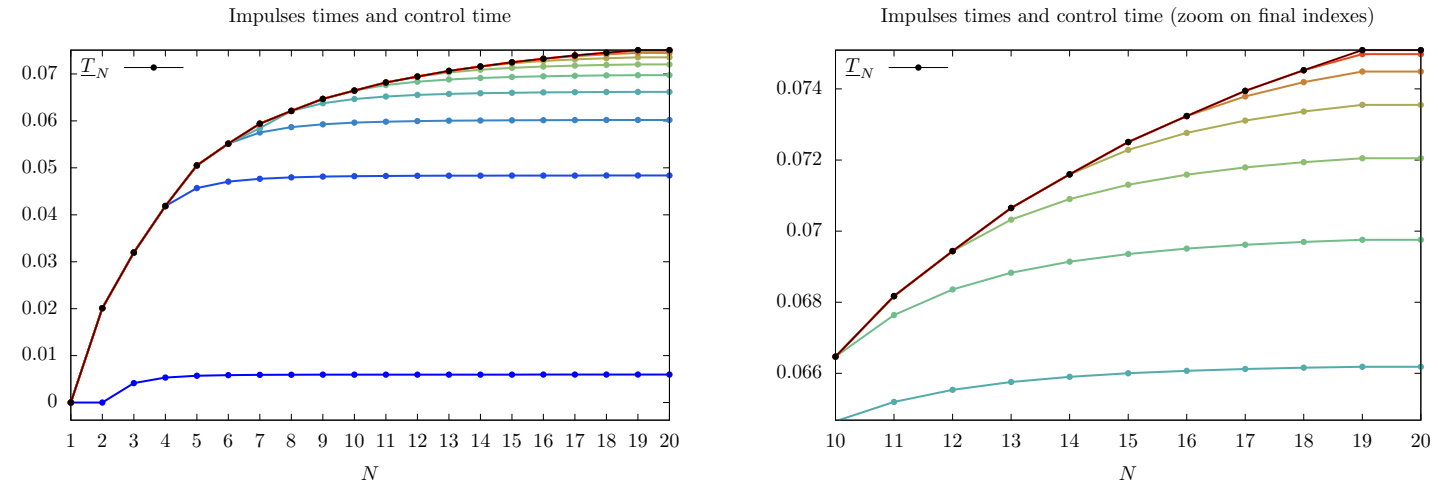

Impulses masses
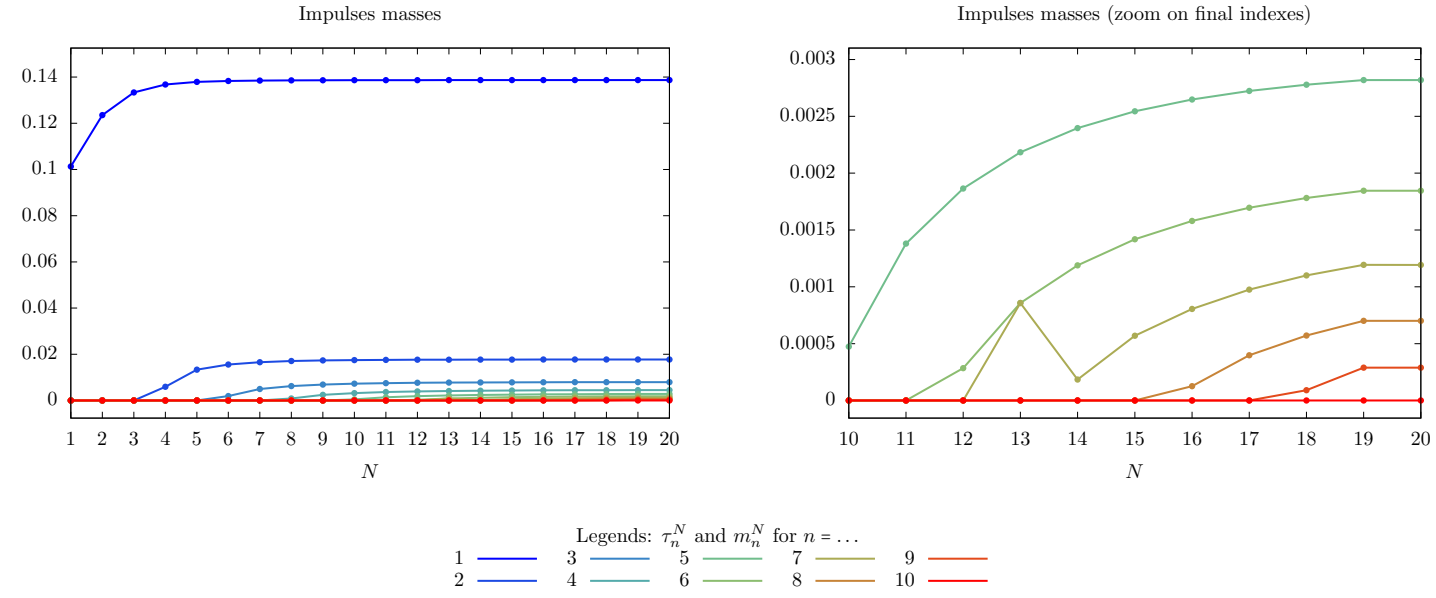

Figure 1: Illustration of the convergence of $\underline{T}_{N}, \tau_{i}^{N}$ and $m_{i}^{N}$ as $N \rightarrow \infty$ for the control system given in Section 4.1

On Figure 2, we display the control obtained for $N=20$ equality constraints. On this figure, we also display the observation $B_{N}^{\top} \psi$ of the adjoint $\psi$. As stated in Lemma 3.1, we observe that $B_{N}^{\top} \psi(t) \geqslant 0$ for every $t \in\left[0, \underline{T}_{N}\right]$, and that Dirac masses are located in the set of times $t \in\left[0, \underline{T}_{N}\right]$ such that $B_{N}^{\top} \varphi(t)=0$. The minimal time obtained with $N=20$ is $\underline{T}_{N} \simeq 0.075091$. We also display on Figure 3 the corresponding state trajectories.

\subsection{Dirichlet 3D spherical heat}

Let us consider the heat equation

$$
\begin{array}{ll}
\dot{\boldsymbol{y}}(t, \boldsymbol{x})=\Delta \boldsymbol{y}(t, \boldsymbol{x}) & (t>0, \boldsymbol{x} \in \Omega), \\
\boldsymbol{y}(t, \boldsymbol{x})=\boldsymbol{u}(t, \boldsymbol{x}) & (t>0, \boldsymbol{x} \in \partial \Omega),
\end{array}
$$


Control impulses and adjoint trace

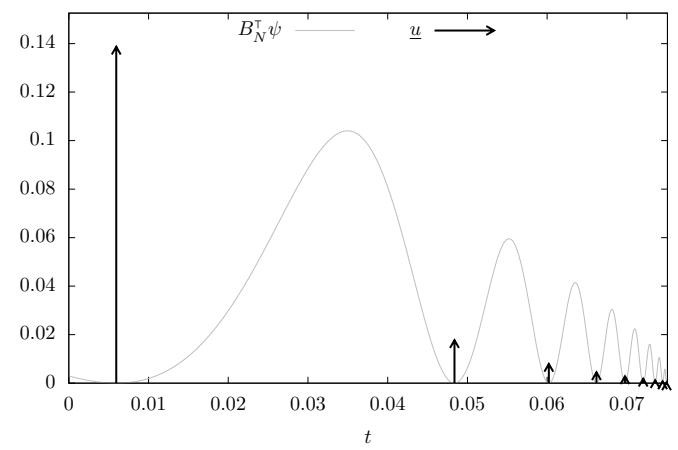

Control impulses and adjoint trace (zoom on final times)

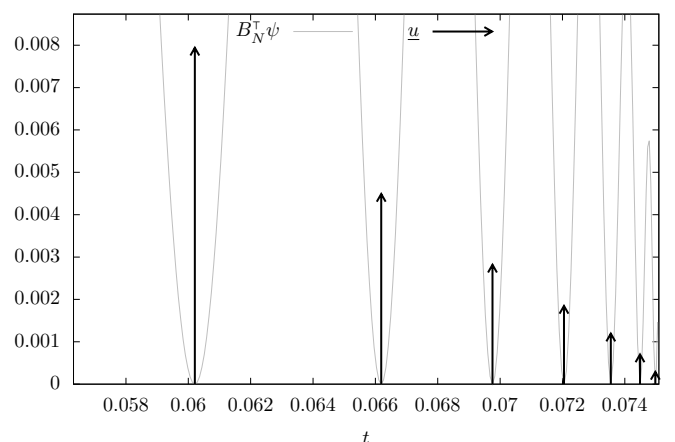

Figure 2: Minimal time control and corresponding adjoint obtained for $N=20$ equality constraints for the system, the initial and target conditions given in Section 4.1. The corresponding state trajectory is given in Figure 3 , and the minimal time obtained is 0.075091 . (Arrows stand for Dirac impulses.)

with $\Omega$ the unit ball of $\mathbb{R}^{3}$. Note that for every $\boldsymbol{y}^{0} \in L^{2}(\Omega)$ and every $\boldsymbol{y}^{1} \in L^{2}(\Omega)$ solution of

$$
\begin{aligned}
\Delta \boldsymbol{y}^{1} & =0 & & \text { in } \Omega, \\
\boldsymbol{y}^{1} & =\overline{\boldsymbol{u}} & & \text { on } \partial \Omega,
\end{aligned}
$$

for some positive $\overline{\boldsymbol{u}} \in L^{2}(\partial \Omega)$, we have, by application of the results contained in 11], that the solution of 4.1) can be steered from $\boldsymbol{y}^{0}$ to $\boldsymbol{y}^{1}$ with a nonnegative control $\boldsymbol{u}$. Furthermore, this requires a minimal time $\underline{\boldsymbol{T}}=\underline{\boldsymbol{T}}\left(\boldsymbol{y}^{0}, \boldsymbol{y}^{1}\right) \geqslant 0$, and there exists a non-negative control $\underline{\boldsymbol{u}} \in \mathcal{M}(\partial \Omega \times$ $[0, \underline{\boldsymbol{T}}])$, steering $\boldsymbol{y}^{0}$ to $\boldsymbol{y}^{1}$ in time $\underline{\boldsymbol{T}}$.

In addition, if $\boldsymbol{y}^{0}$ and $\boldsymbol{y}^{1}$ are radially symmetric, i.e., $\boldsymbol{y}^{0}(\boldsymbol{x})=y^{0}(|\boldsymbol{x}|)$ and $\boldsymbol{y}^{1}(\boldsymbol{x})=y^{1}(|\boldsymbol{x}|)$, then the control $\underline{\boldsymbol{u}}$ can be chosen radially symmetric, i.e., $\underline{\boldsymbol{u}}(t, \boldsymbol{x})=\underline{u}(t)$. We then have that $\boldsymbol{y}(t, \cdot)$ is radially symmetric for every $t$, and $y$ defined by $y(t,|\boldsymbol{x}|)=\boldsymbol{y}(t, x)$ is solution of

$$
\begin{aligned}
\dot{y}(t, x) & =\frac{1}{x^{2}} \partial_{x}\left(x^{2} \partial_{x} y(t, x)\right) & & (t>0, x \in(0,1)), \\
\partial_{x} y(t, 0) & =0 & & (t>0), \\
y(t, 1) & =u(t) & & (t>0), \\
y(0, x) & =y^{0}(x) & & (x \in(0,1)) .
\end{aligned}
$$

Even if 4.2 does not fit the requirement made for 1.1), it is however classical that the solution of 4.2 can be decomposed as

$$
y(t, x)=\sum_{n=1}^{\infty} a_{n} e^{-\lambda_{n} t} \varphi_{n}(x)+\sum_{n=1}^{\infty} \gamma_{n} \int_{0}^{t} e^{-\lambda_{n}(t-s)} u(t) \mathrm{d} t \varphi_{n}(x),
$$

where for every $n \in \mathbb{N}^{*}$, we have set

$$
\lambda_{n}=-(n \pi)^{2}, \quad \varphi_{n}(x)=\sqrt{2} n \pi \operatorname{sinc}(n \pi x) \quad(x \in[0,1]) \quad \text { and } \quad \gamma_{n}=-\partial_{x} \varphi_{n}(1)=(-1)^{n+1} \sqrt{2} n \pi .
$$

In addition, it is classical that the operator $L$ defined by

$$
\begin{aligned}
& \mathcal{D}(L)=\left\{y \in H_{x^{2}}^{2}(0,1) \mid \partial_{x} y(0)=y(1)=0\right\}, \\
& L y=\left(x \in(0,1) \mapsto \frac{1}{x^{2}} \partial\left(x^{2} \partial_{x} y(x) \in \mathbb{R}\right)\right) \in L_{x^{2}}^{2}(0,1) \quad(y \in \mathcal{D}(L)),
\end{aligned}
$$



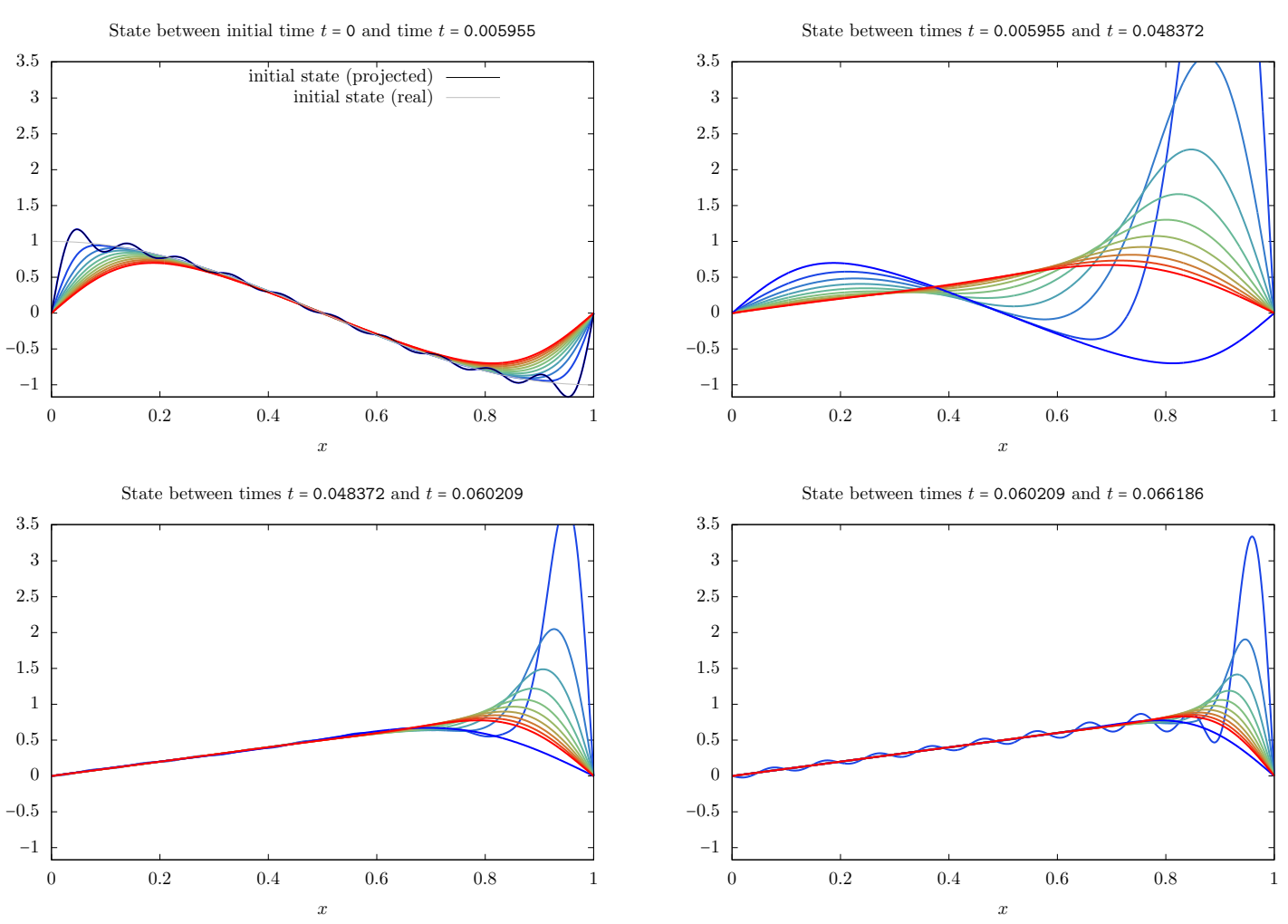

State between times $t=0.066186$ and $t=0.069755$

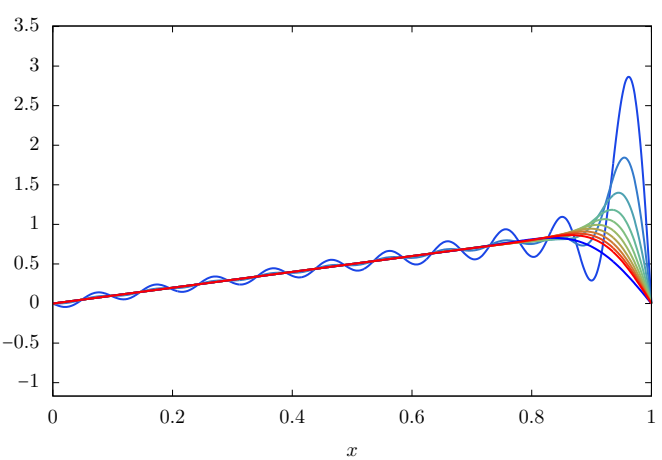

State between times $t=0.069755$ and final time $t=0.075091$

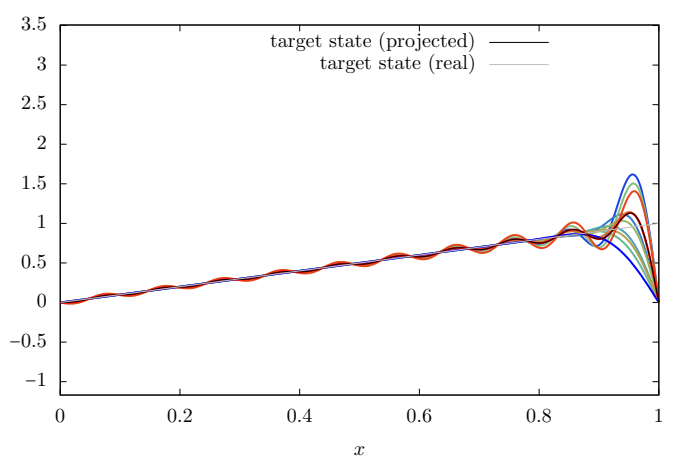

Figure 3: State trajectory obtained for $N=20$ equality constraints for the system, the initial and target conditions given in Section 4.1. The corresponding minimal time control is given in Figure 2, and the minimal time obtained is 0.075091 . (On each plot, the color goes from blue (the state at the minimal time of the plot) to red (the state at the maximal time of the plot). The initial (respectively target) projected state is $\sum_{n=1}^{N} Y_{n}^{i} \varphi_{n}$, for $i=0$ (respectively $\left.i=1\right)$.)

is self-adjoint for the scalar product $(y, z)=\int_{0}^{1} y(x) z(x) x^{2} \mathrm{~d} x$. In the above definition, we have used $L_{x^{2}}^{2}(0,1)=\left\{y:\left.(0,1) \rightarrow \mathbb{R}\left|\int_{0}^{1}\right| y(x)\right|^{2} x^{2} \mathrm{~d} x<\infty\right\}$ and $H_{x^{2}}^{2}(0,1)=\{y:(0,1) \rightarrow \mathbb{R} \mid$ $\partial_{x} y \in L_{x^{2}}^{2}(0,1)$ and $\left.\partial_{x}^{2} y \in L_{x^{2}}^{2}(0,1)\right\}$. One can also check that $\left\{\varphi_{n}\right\}_{n \in \mathbb{N}^{*}}$ is an orthonormal basis of $L_{x^{2}}^{2}(0,1)$. In addition, one can see that the set of positive steady states of 4.2 is $\mathcal{S}_{+}^{*}=\left\{x \in[0,1] \mapsto v \mid v \in \mathbb{R}_{+}^{*}\right\}$. With this operator $L$, one can also check that the conclusions of 
Theorem 1 also holds. In particular, for every $y^{0} \in L_{x^{2}}^{2}(0,1)$ and every $y^{1} \in \mathcal{S}_{+}^{*}$, there exists a minimal time $\underline{T}\left(y^{0}, y^{1}\right)$ required to steer $y^{0}$ to $y^{1}$ with nonnegative controls. Furthermore, at time $\underline{T}\left(y^{0}, y^{1}\right)$, there exists a nonnegative purely impulsive control $\underline{u}$ steering $y^{0}$ to $y^{1}$ in time $\underline{T}\left(y^{0}, y^{1}\right)$ and this control can be approximated by sequence of minimizers of $(3.3)$.

For the numerical simulation, we consider $y^{1}(x)=1 \in \mathcal{S}_{+}^{*}$ and $y^{0}(x)=\cos (\pi x) \in L_{x^{2}}^{2}(0,1)$ and we have,

$$
\begin{aligned}
Y_{n}^{0}=\int_{0}^{1} y^{0}(x) \varphi_{n}(x) x^{2} \mathrm{~d} x=\left\{\begin{array}{ll}
\frac{-1}{2 \sqrt{2} \pi} & \text { if } n=1, \\
\frac{(-1)^{n} \sqrt{2} n}{\left(n^{2}-1\right) \pi} & \text { otherwise }
\end{array} \quad\right. \text { and } \\
Y_{n}^{1}=\int_{0}^{1} y^{1}(x) \varphi_{n}(x) x^{2} \mathrm{~d} x=\frac{(-1)^{n+1} \sqrt{2}}{n \pi} \quad\left(n \in \mathbb{N}^{*}\right) .
\end{aligned}
$$

Here also, in order to numerically solve $(3.3)$, we use the sequential quadratic programming method of the optimization toolbox of matlab. On Figure 4 , we display the values of $\underline{T}_{N}=\underline{T}_{N}\left(y^{0}, y^{1}\right)$, $\tau_{i}^{N}$ and $m_{i}^{N}$ with respect to $N$. Recall that for some given $N \in \mathbb{N}^{*}$, the optimal control of 3.3 is a some of at most $\lfloor(N+1) / 2\rfloor$ Dirac masses and for $i>\lfloor(N+1) / 2\rfloor$, we have set $\tau_{i}^{N}=\underline{T}_{N}$ and $m_{i}^{N}=0$.

On Figure 5, we display the control obtained for $N=20$ equality constraints. On this figure, we also display the observation $B_{N}^{\top} \psi$ of the adjoint $\psi$. As stated in Lemma 3.1. we observe that $B_{N}^{\top} \psi(t) \geqslant 0$ for every $t \in\left[0, \underline{T}_{N}\right]$, and that Dirac masses are located in the set of times $t \in\left[0, \underline{T}_{N}\right]$ such that $B_{N}^{\top} \varphi(t)=0$. The minimal time obtained with $N=20$ is $\underline{T}_{N} \simeq 0.103882$. We also display on Figure 6 the corresponding state trajectories.

\subsection{Coupled heat system}

For this example we consider the coupled heat equation given by

$$
\begin{aligned}
\dot{y}_{1}(t, x) & =\partial_{x}^{2} y_{1}(t, x)+y_{2}(t, x) & & (t>0, x \in(0,1)), \\
\dot{y}_{2}(t, x) & =\partial_{x}^{2} y_{2}(t, x) & & (t>0, x \in(0,1)), \\
y_{1}(t, 0) & =y_{1}(t, 1)=y_{2}(t, 0)=0 & & (t>0), \\
y_{2}(t, 1) & =u(t) & & (t>0), \\
y_{1}(0, x) & =y_{1}^{0}(x) & & (x \in(0,1), \\
y_{2}(0, x) & =y_{2}^{0}(x) & & (x \in(0,1) .
\end{aligned}
$$

For this system, we observe that the set of positive steady states is given by

$$
\mathcal{S}_{+}^{*}=\left\{x \in[0,1] \mapsto\left(u x\left(1-x^{2}\right), 6 u x\right) \mid u \in \mathbb{R}_{+}^{*}\right\} .
$$

Let us also define the operator $L$ by

$$
\begin{aligned}
& \mathcal{D}(L)=\left(H^{2}(0,1) \cap H_{0}^{1}(0,1)\right) \times\left(H^{2}(0,1) \cap H_{0}^{1}(0,1)\right), \\
& L\left(y_{1}, y_{2}\right)=\left(\partial_{x}^{2} y_{1}+y_{2}, \partial_{x}^{2} y_{2}\right) \quad\left(y_{1}, y_{2}\right) \in \mathcal{D}(L) .
\end{aligned}
$$

One can see that $L$ is $m$-dissipative. In addition, according to 8 , the system 4.3 is null controllable. However, it is not proved that for every $y_{1}^{0}, y_{2}^{0} \in L^{2}(0,1)$, the system is null controllable 
Impulses times and control time

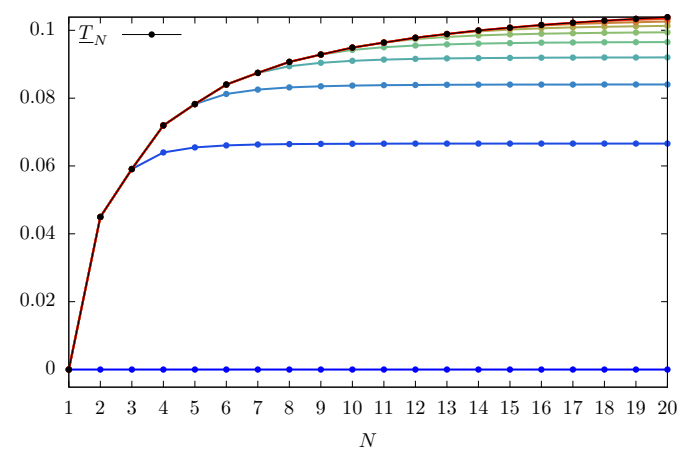

Impulses masses

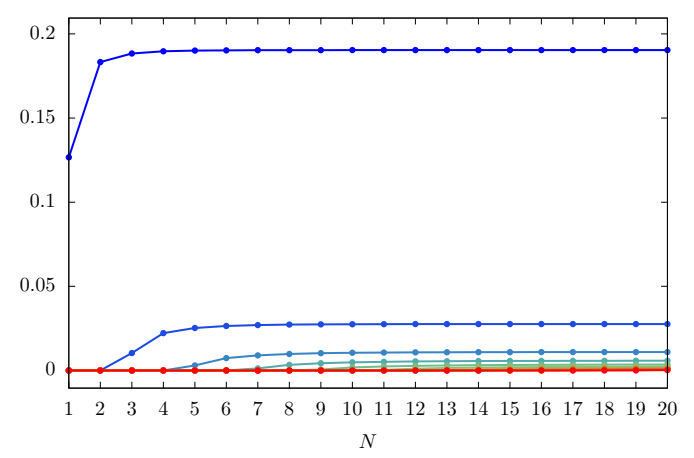

Impulses times and control time (zoom on final indexes)

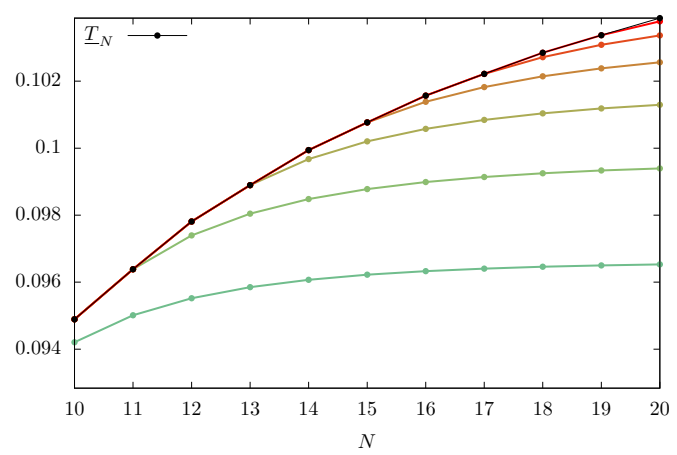

Impulses masses (zoom on final indexes)

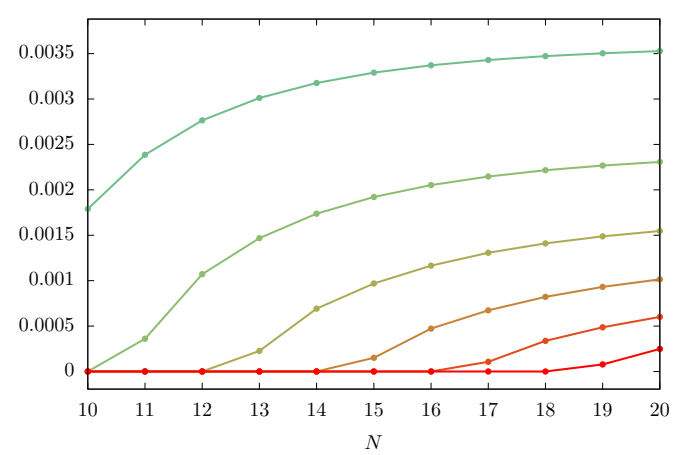

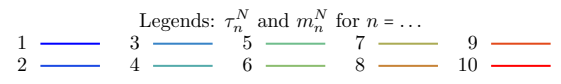

Figure 4: Illustration of the convergence of $\underline{T}_{N}, \tau_{i}^{N}$ and $m_{i}^{N}$ as $N \rightarrow \infty$ for the control system given in Section 4.2 .
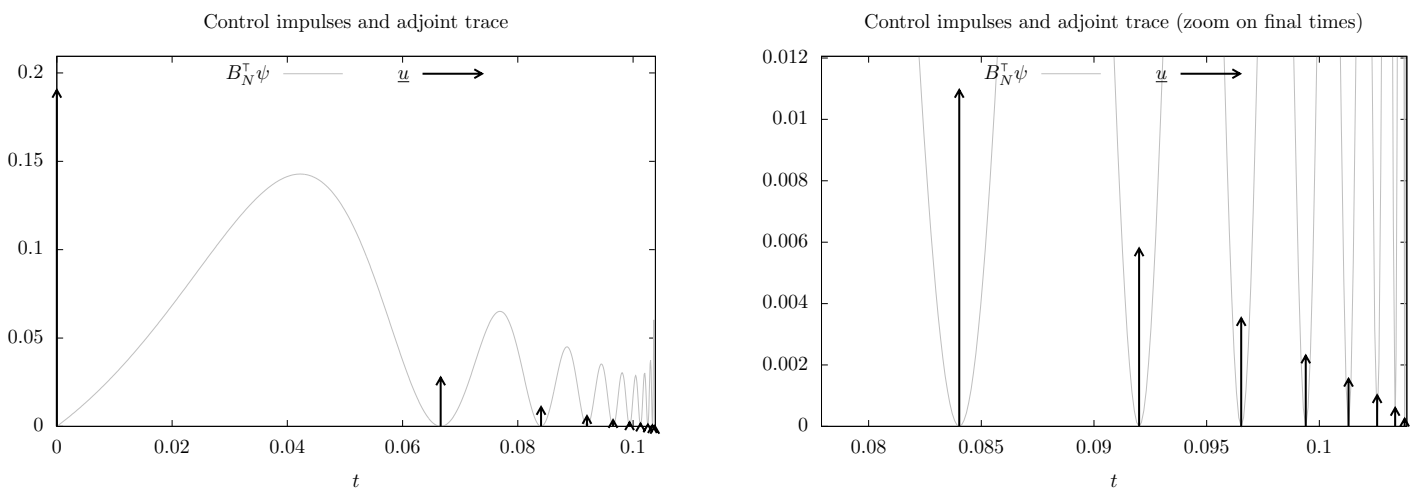

Figure 5: Minimal time control and corresponding adjoint obtained for $N=20$ equality constraints for the system, the initial and target conditions given in Section 4.2. The corresponding state trajectory is given in Figure 6, and the minimal time obtained is 0.103882. (Arrows stand for Dirac impulses.) 

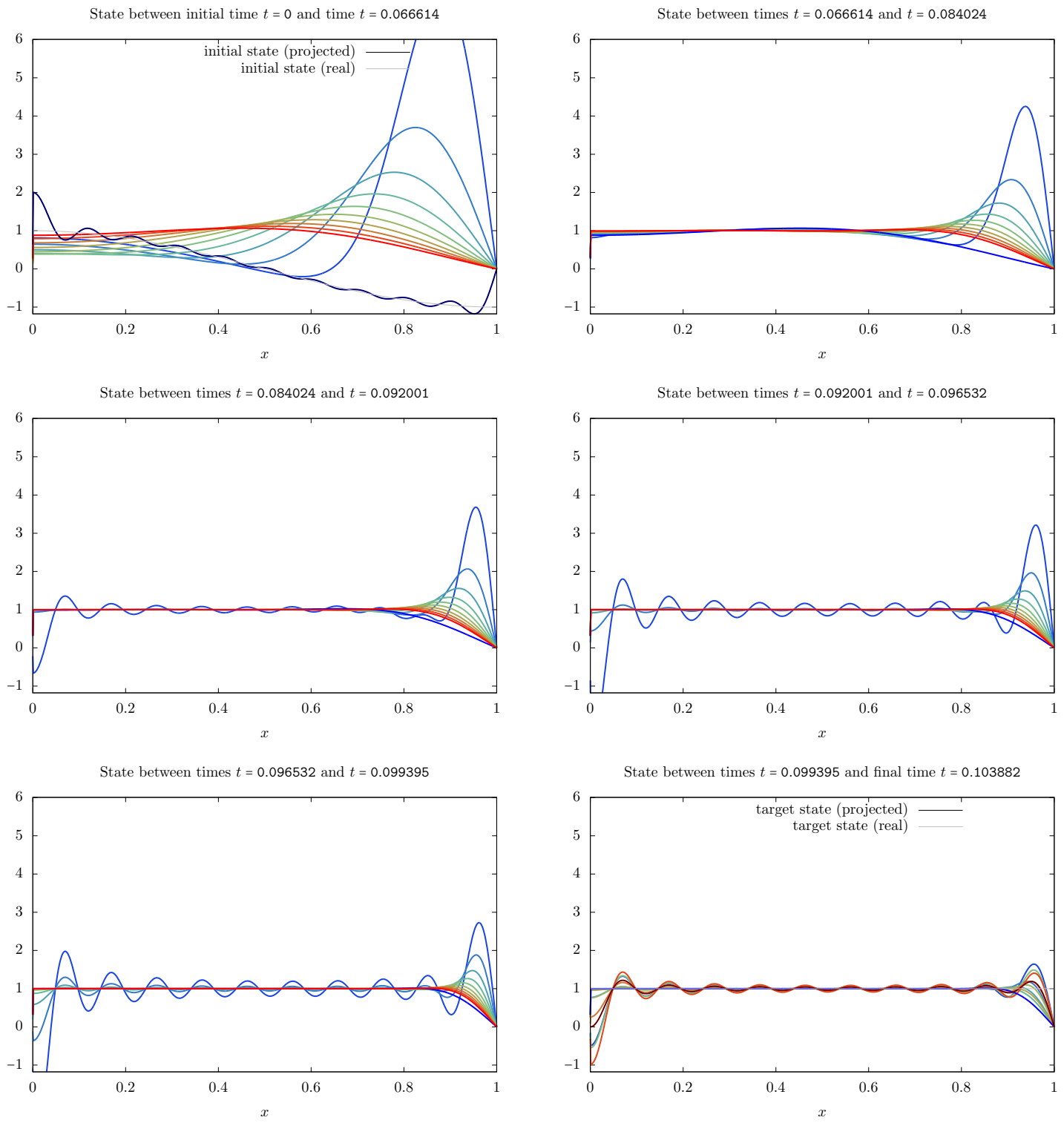

Figure 6: State trajectory obtained for $N=20$ equality constraints for the system, the initial and target conditions given in Section 4.2. The corresponding minimal time control is given in Figure 5 , and the minimal time obtained is 0.103882 . (On each plot, the color goes from blue (the state at the minimal time of the plot) to red (the state at the maximal time of the plot). The initial (respectively target) projected state is $\sum_{n=1}^{N} Y_{n}^{i} \varphi_{n}$, for $i=0$ (respectively $\left.i=1\right)$.)

with continuous controls. This fact is required to prove the existence of a time $T>0$ and a nonnegative control $u \in L^{2}(0, T)$ steering the solution of (4.3) from $y^{0}=\left(y_{1}^{0}, y_{2}^{0}\right) \in L^{2}(0,1) \times L^{2}(0,1)$ to $y^{1}=\left(y_{1}^{1}, y_{1}^{1}\right) \in \mathcal{S}_{+}^{*}$ in time $T$. In other words, this fact is required to prove that $\underline{T}\left(y^{0}, y^{1}\right)<\infty$.

Note also that the operator $L$ is not diagonalizable. However, defining for every $n \in \mathbb{N}^{*}, \varphi_{n}$ 
and $\lambda_{n}$ as in Section 4.1, we observe that

$$
L\left(\varphi_{n}, 0\right)=-\lambda_{n}\left(\varphi_{n}, 0\right) \quad \text { and } \quad L\left(0, \varphi_{n}\right)=-\lambda_{n}\left(0, \varphi_{n}\right)+\left(\varphi_{n}, 0\right) .
$$

It is also trivial that $\left\{\left(\varphi_{n}, 0\right)\right\}_{n \in \mathbb{N}^{*}} \cup\left\{\left(0, \varphi_{n}\right)\right\}_{n \in \mathbb{N}^{*}}$ is an orthonormal basis of $L^{2}(0,1) \times L^{2}(0,1)$.

As previously, if $\underline{u} \in \mathcal{M}([0, T])$ is a control steering some initial condition $y^{0}=\left(y_{1}^{0}, y_{2}^{0}\right)$ to a target $y^{1}=\left(y_{1}^{1}, y_{2}^{1}\right)$ in some time $T>0$, then we have, for every $n \in \mathbb{N}^{*}$,

$$
\begin{aligned}
Y_{1, n}^{1}-e^{-\lambda_{n} T}\left(Y_{1, n}^{0}+T Y_{2, n}^{0}\right) & =\gamma_{n} \int_{0}^{T}(T-t) e^{-\lambda_{n}(T-t)} \mathrm{d} \underline{u}(t) \\
Y_{2, n}^{1}-e^{-\lambda_{n} T} Y_{2, n}^{0} & =\gamma_{n} \int_{0}^{T} e^{-\lambda_{n}(T-t)} \mathrm{d} \underline{u}(t) .
\end{aligned}
$$

where for every $n \in \mathbb{N}^{*}, Y_{k, n}^{i}=\int_{0}^{1} y_{k}^{i}(x) \varphi_{n}(x) \mathrm{d} x$ (with $i \in\{0,1\}$ and $k \in\{1,2\}$ ), and $\gamma_{n}$ is defined as in Section 4.1. In particular, for an impulsive control, $\underline{u}=\sum_{i=1}^{\infty} m_{i} \delta_{\tau_{i}}$, we have,

$$
\begin{aligned}
Y_{1, n}^{1}-e^{-\lambda_{n} T}\left(Y_{1, n}^{0}+T Y_{2, n}^{0}\right) & =\gamma_{n} \sum_{i=0}^{\infty} m_{i}\left(T-\tau_{i}\right) e^{-\lambda\left(T-\tau_{i}\right)}, \\
Y_{2, n}^{1}-e^{-\lambda_{n} T} Y_{2, n}^{0} & =\gamma_{n} \sum_{i=0}^{\infty} m_{i} e^{-\lambda_{n}\left(T-\tau_{i}\right)} .
\end{aligned}
$$

Assuming that null controllability of 4.3 holds with continuous controls, one can repeat the development made in Section 3 to conclude that similar result hold. In particular, for every $y^{0}=\left(y_{1}^{0}, y_{2}^{0}\right) \in L^{2}(0,1) \times L^{2}(0,1)$ and every $y^{1}=\left(y_{1}^{1}, y_{2}^{1}\right) \in \mathcal{S}_{+}^{*}$, there exists a minimal time $\underline{T}\left(y^{0}, y^{1}\right)$ required to steer $y^{0}$ to $y^{1}$ with nonnegative controls. Furthermore, at time $\underline{T}\left(y^{0}, y^{1}\right)$, there exists a nonnegative purely impulsive control $\underline{u}$ steering $y^{0}$ to $y^{1}$ in time $\underline{T}\left(y^{0}, y^{1}\right)$ and this control can be approximated, as $N \rightarrow \infty$, by sequence of minimizers of the minimization problem

$$
\begin{aligned}
& \underline{T}_{N}\left(y^{0}, y^{1}\right)=\min T \\
& \qquad \begin{array}{l}
T \geqslant 0, \\
\exists \underline{u} \in \mathcal{M}([0, T]) \text { s.t. } \underline{u} \geqslant 0 \text { and } \forall n \in\{1, \ldots, N\} . \\
\\
Y_{1, n}^{1}-e^{-\lambda_{n} T}\left(Y_{1, n}^{0}+T Y_{2, n}^{0}\right)=\gamma_{n} \sum_{i=0}^{\infty} m_{i}\left(T-\tau_{i}\right) e^{-\lambda\left(T-\tau_{i}\right)} \text { and } \\
\quad Y_{2, n}^{1}-e^{-\lambda_{n} T} Y_{2, n}^{0}=\gamma_{n} \sum_{i=0}^{\infty} m_{i} e^{-\lambda_{n}\left(T-\tau_{i}\right)} .
\end{array}
\end{aligned}
$$

Note that applying the results of [12, we obtain that the control minimizing (4.4) is a sum of at most $N$ Dirac masses.

For the numerical example, we consider $y_{1}^{1}(x)=x\left(1-x^{2}\right), y_{2}^{1}(x)=6 x, y_{1}^{0}(x)=-x$ and $y_{2}^{0}(x)=$ $\cos (\pi x)$, we then have, for every $n \in \mathbb{N}^{*}$,

$$
\begin{array}{lrl}
Y_{1, n}^{0}=\frac{(-1)^{n} \sqrt{2}}{n \pi}, & Y_{1, n}^{1}=\frac{(-1)^{n+1} 6 \sqrt{2}}{(n \pi)^{3}}, \\
Y_{2, n}^{0}=\frac{\left(1+(-1)^{n}\right) \sqrt{2} n}{\left(n^{2}-1\right) \pi}, & Y_{2, n}^{1}=\frac{(-1)^{n+1} 6 \sqrt{2}}{n \pi} .
\end{array}
$$

As for the previous examples, in order to numerically solve (3.3), we use the sequential quadratic programming method of the optimization toolbox of matlab. On Figure7, we display the values of $\underline{T}_{N}=\underline{T}_{N}\left(y^{0}, y^{1}\right), \tau_{i}^{N}$ and $m_{i}^{N}$ with respect to $N$. Recall that for some given $N \in \mathbb{N}^{*}$, the optimal 
Impulses times and control time

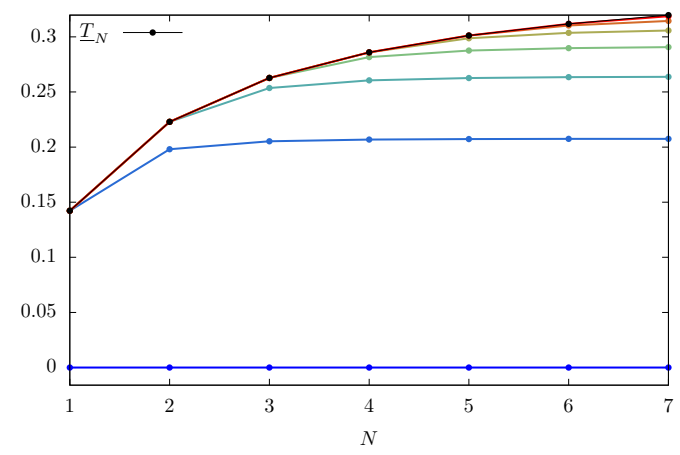

Impulses masses

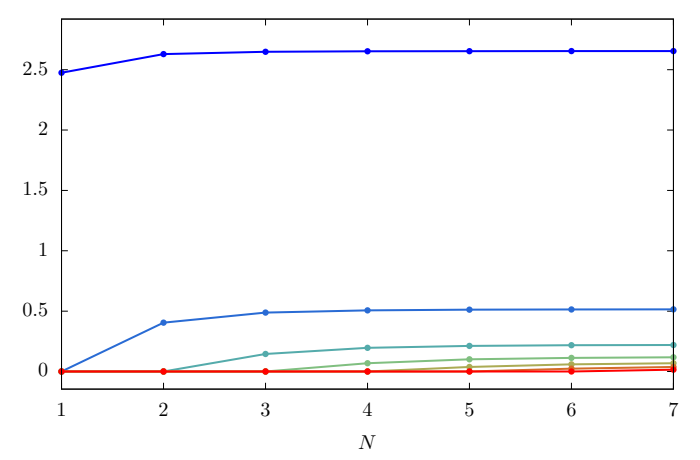

Impulses times and control time (zoom on final indexes)

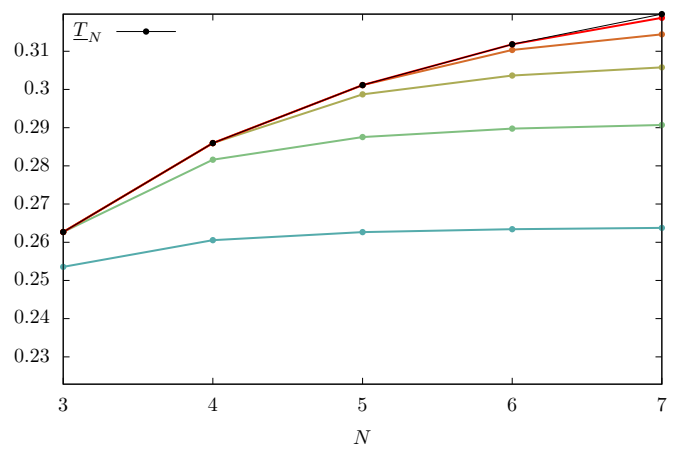

Impulses masses (zoom on final indexes)

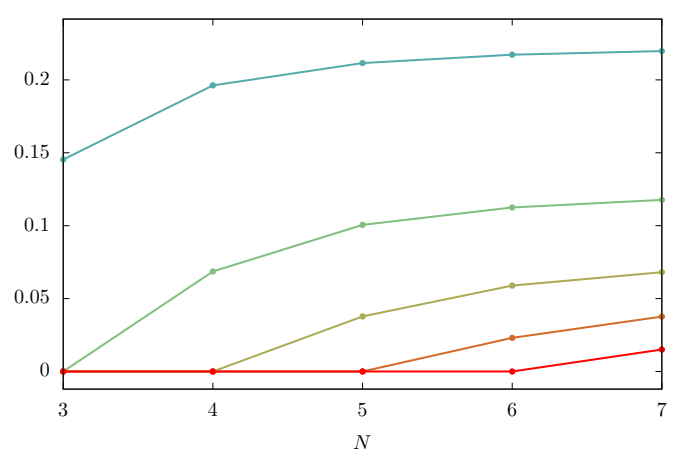

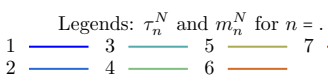

Figure 7: Illustration of the convergence of $\underline{T}_{N}, \tau_{i}^{N}$ and $m_{i}^{N}$ as $N \rightarrow \infty$ for the control system given in Section 4.3

control of (3.3) is a sum of at most $N$ Dirac masses and for $i>N$, we have set $\tau_{i}^{N}=\underline{T}_{N}$ and $m_{i}^{N}=0$.

On Figure 8, we display the control obtained for $N=7$ equality constraints (unfortunately, I did not succeed to have convergence to a minimizer of (4.4) for $N>7$ ). On this figure, we also display the observation $B_{N}^{\top} \psi$ of the adjoint $\psi$. As for the previous examples, we observe that the adjoint observation is of constant sign, and that Dirac masses are located on times when the adjoint observation vanishes (these facts were expected from [12]). The minimal time obtained with $N=7$ is $\underline{T}_{N} \simeq 0.319723$. We also display on Figures 9 and 10 the corresponding state trajectories.

\section{Conclusion and open questions}

In this paper, we have shown, for one dimensional heat equation, that steering an initial state to some positive target state with a nonnegative control requires some minimal time $\underline{T}$. At this minimal controllability time $\underline{T}$, there exists a nonnegative control, steering the initial state to the target state in time $\underline{T}$, in the space of Radon measures which is a countable sum of Dirac masses. In 

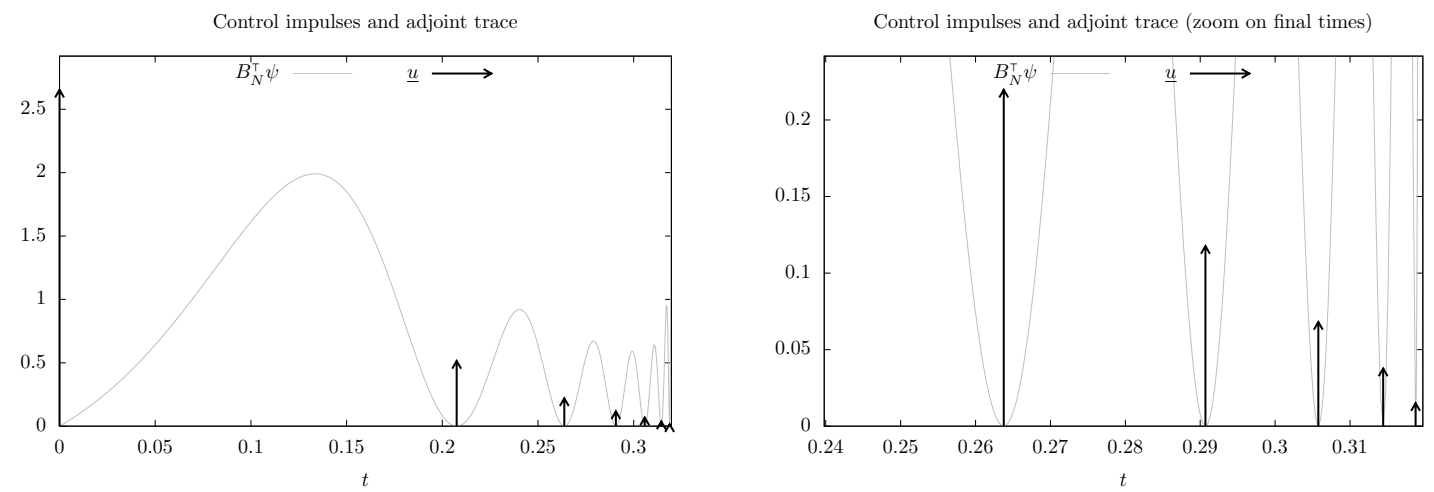

Figure 8: Minimal time control and corresponding adjoint obtained for $N=7$ equality constraints for the system, the initial and target conditions given in Section 4.3 The corresponding state trajectory is given in Figures 9 and 10 and the minimal time obtained is 0.319723 . (Arrows stand for Dirac impulses.)

addition, the minimal time $\underline{T}$ and the associated purely impulsive control can be obtained through a sequence of minimization problems of finite dimension.

Some open questions are listed below.

Uniqueness of the minimal time control. It has been shown that the minimal time control is unique in the space of purely impulsive controls. It remains to show that any control at the minimal time do not have a continuous part. This will give the uniqueness of the minimal time control in the space of Radon measures. If one aim to follow the strategy of the present paper, to prove that the constructed control is unique in the space of Radon measures, one shall extend Lemma 3.6 to the following statement.

$$
\begin{aligned}
& \text { Let } T>0, v \in \mathcal{M}([0, T]) \text { and }\left(\theta_{k}\right)_{k \in \mathbb{N}^{*}} \in([0, T])^{N^{*}} \text {. Assume that } \theta_{k} \neq \theta_{j} \text { for every } j \neq k \\
& \text { and that } v(\{t\})=0 \text { for every } t \in[0, T] \text {. Then, the family }\left\{\left(\gamma_{n} \int_{[0, T]} e^{-\lambda_{n}(T-t)} \mathrm{d} v(t)\right)_{n \in \mathbb{N}^{*}}\right\} \cup \\
& \left\{\left(\gamma_{n} e^{-\lambda_{n}\left(T-\theta_{k}\right)}\right)_{n \in \mathbb{N}^{*}}\right\}_{k \in \mathbb{N}^{*}} \text { is free in } \mathbb{R}^{N^{*}} \text {. }
\end{aligned}
$$

Adjoint state at the minimal control time. For the finite dimensional system (3.3), it has been shown in $\left[12\right.$ that at the minimal time $\underline{T}_{N}$, there exists an adjoint state $\psi^{N}$ such that its observation $B_{N}^{\top} \psi^{N}$ has a constant sign on $\left[0, \underline{T}_{N}\right]$, and such that the time impulses of the minimal time control are located in the set of times when the adjoint observation vanishes. Such a result has not been proved for the continuous heat equation. If we aim to prove such a result, by passing to the limit $N \rightarrow \infty$, we need some compactness properties on the sequence $\left(\psi^{N}\left(\underline{T}_{N}\right)\right)_{N}$. A better understanding of relation between adjoint observation and minimal time control would be nice, in particular for developing more powerful numerical methods.

Convergence rate. We have seen that the minimal time $\underline{T}_{N}$ and minimal time control $\underline{u}^{N}$ minimizers of (3.3) converges to the minimal time and control $\underline{T}$ and $\underline{u}$, minimizers of (3.1). Estimations of the convergence rates are unknown.

Coupled heat system. For the coupled heat system considered in Section 4.3 , it has been seen that Theorem 1 also applies, provided that null controllability of (4.3) could be achieved with the help of continuous controls. As far as I know, this is an open problem. 
State $y_{1}$ between initial time $t=0$ and time $t=0.207483$

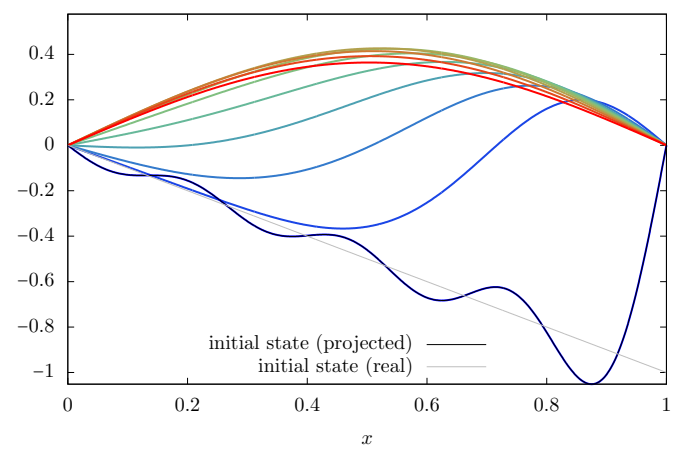

State $y_{1}$ between times $t=0.207483$ and $t=0.263758$

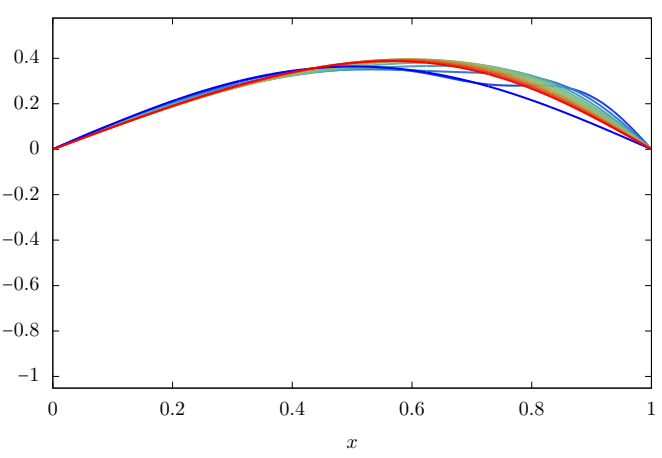

State $y_{1}$ between times $t=0.263758$ and $t=0.290691$

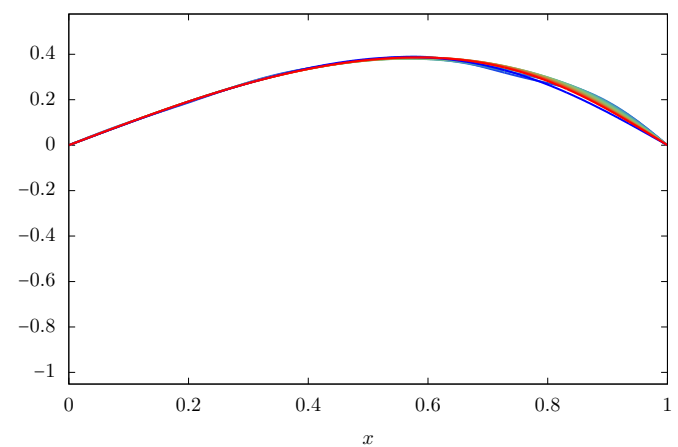

State $y_{2}$ between initial time $t=0$ and time $t=0.207483$

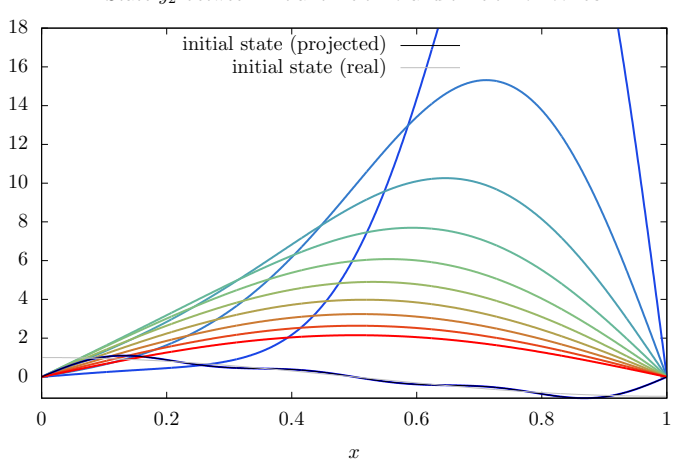

State $y_{2}$ between times $t=0.207483$ and $t=0.263758$

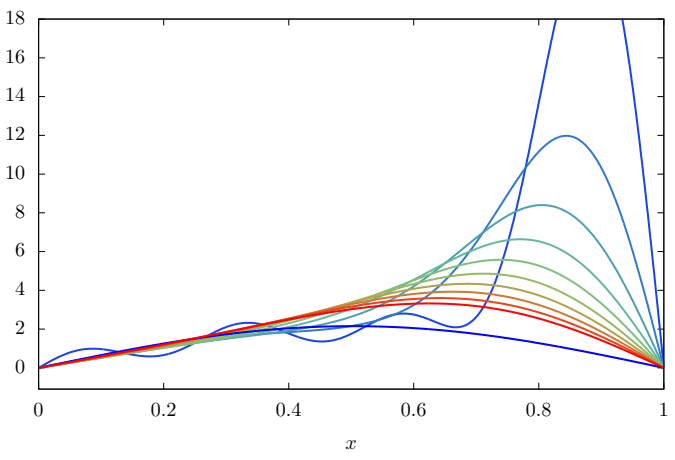

State $y_{2}$ between times $t=0.263758$ and $t=0.290691$

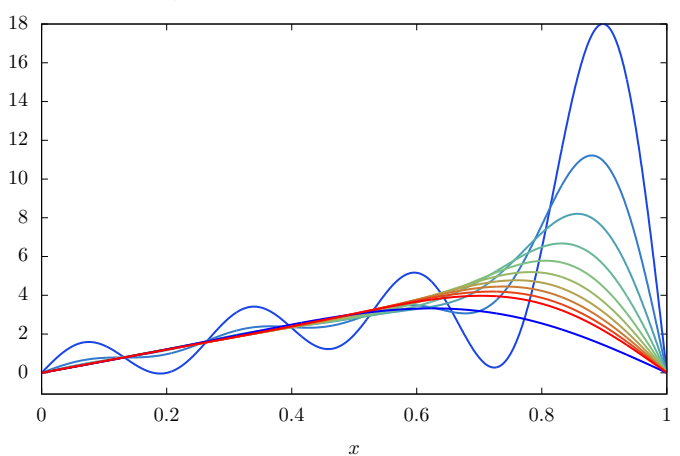

Figure 9: State trajectory ( $y_{1}$ left and $y_{2}$ right) obtained for $N=7$ equality constraints for the system, the initial and target conditions given in Section 4.3 . The corresponding minimal time control is given in Figure 8, and the minimal time obtained is 0.319723 . The state for final times is displayed in Figure 10. (On each plot, the color goes from blue (the state at the minimal time of the plot) to red (the state at the maximal time of the plot). The initial (respectively target) projected state is $\sum_{n=1}^{N} Y_{j, n}^{i} n \varphi_{n}$, for $i=0$ (respectively $i=1$ ) and for $j \in\{1,2\}$.) 
State $y_{1}$ between times $t=0.290691$ and $t=0.305769$

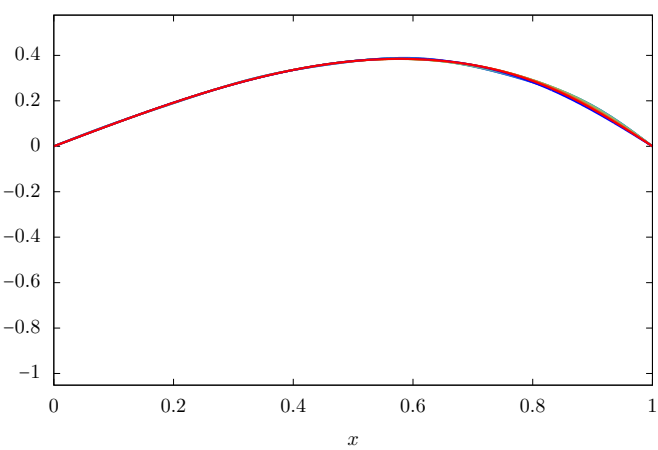

State $y_{1}$ between times $t=0.305769$ and $t=0.314420$

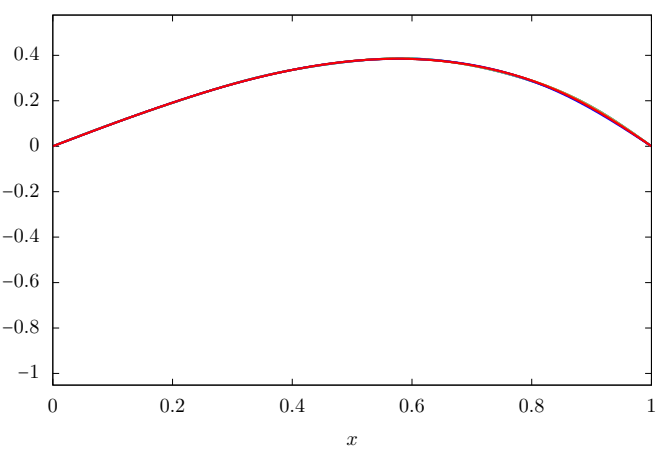

State $y_{1}$ between times $t=0.314420$ and final time $t=0.319723$

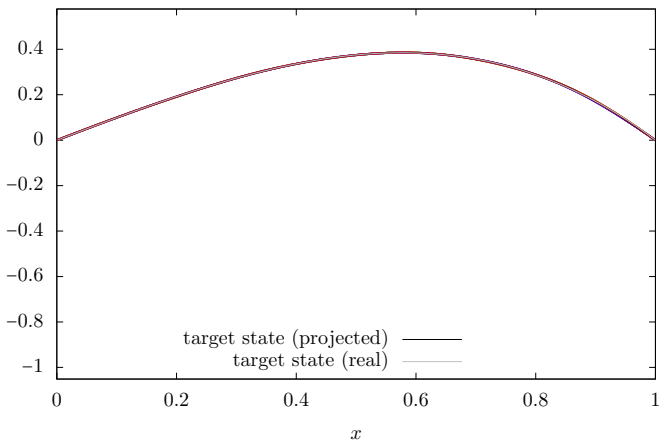

State $y_{2}$ between times $t=0.290691$ and $t=0.305769$

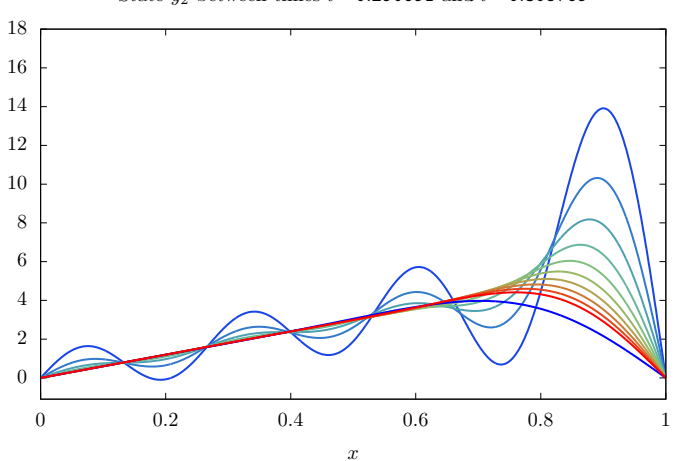

State $y_{2}$ between times $t=0.305769$ and $t=0.314420$

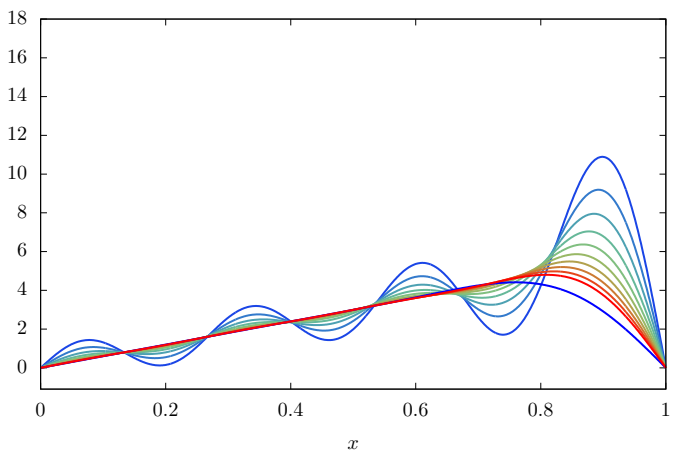

State $y_{2}$ between times $t=0.314420$ and final time $t=0.319723$

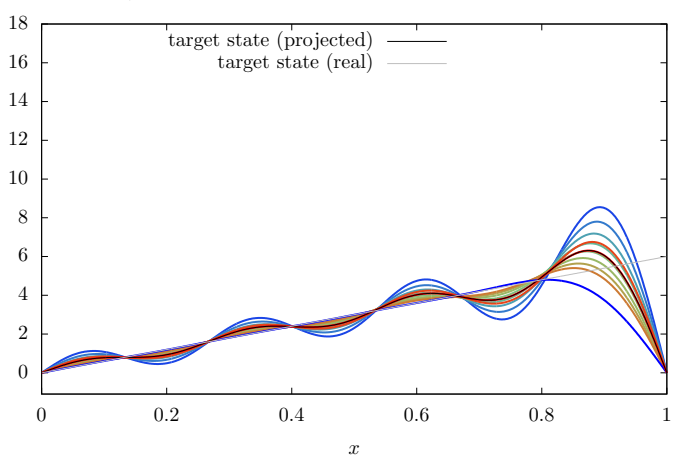

Figure 10: Figure 9 continued.

Approximate controllability. The problem consider in this article can be also stated for approximate controllability. That it to say, for $\varepsilon>0$, find

$$
\begin{aligned}
& \underline{T}^{\varepsilon}\left(y^{0}, y^{1}\right)=\inf \left\{T>0 \mid \exists u \in L^{2}(0, T) \text { s.t. } u \geqslant 0\right. \\
& \left.\quad \text { and the solution } y \text { of } 1.1 \text { satisfies }\left\|y^{1}-y(T, \cdot)\right\|_{L^{2}(0,1)} \leqslant \varepsilon\right\},
\end{aligned}
$$

Needless to say that $\underline{T}^{\varepsilon}\left(y^{0}, y^{1}\right) \leqslant \underline{T}\left(y^{0}, y^{1}\right)$. For $\varepsilon>0$ small enough (the smallness depends on $y^{0}$ and $\left.y^{1}\right)$, one can similarly show that if $y^{0} \neq y^{1}$, then $\underline{T}^{\varepsilon}\left(y^{0}, y^{1}\right)>0$, in addition in the minimal time $\underline{T}^{\varepsilon}\left(y^{0}, y^{1}\right)$ there exist a nonnegative control in the space of Radon measures steering $y^{0}$ at a distance $\varepsilon$ of $y^{1}$ in time $\underline{T}^{\varepsilon}\left(y^{0}, y^{1}\right)$. To prove this fact, one can observe that all moments $Y_{n}^{0}$ 
have to be steered to the moments $Y_{n}^{1}$ at a distance $\varepsilon$, and we can procede as in Appendix B. In Theorem 1, we have stated that a control purely impulsive control steering $y^{0}$ to $y^{1}$ is composed of an infinite number of Dirac masses. As far as I know this property is open for approximate controllability.

Heat equation in higher dimension. The result of this paper are mainly based on the results of 12, where a scalar control is considered. With such constraint one can for instance consider the heat equation with strong symmetry properties, as it has been done in Section 4.2 . Note that it shall not be a problem to obtain the results of 12 for multidimensional inputs. This would allow considering for instance the 1D heat equation with controls at both ends.

For general multi-dimensional heat equation, it has been proved in [11, that a Dirichlet control exist in the minimal controllability time, and this control is also a nonnegative Radon measure. But this control depends on time and space, hence, proving that this control is purely impulsive is much more difficult.

Estimation of the minimal time. Some lower bound on the minimal time have been obtained in [11, however as already mentioned in this paper, these lower bounds are not optimal. Note also that $\underline{T}_{N}\left(y^{0}, y^{1}\right)$ is a lower bound on the minimal time $\underline{T}\left(y^{0}, y^{1}\right)$. But, we do not have a priori estimates on the time $\underline{T}_{N}\left(y^{0}, y^{1}\right)$.

\section{A Controllability with nonnegative controls}

Let us first recall that, according to [7, for every $y^{0} \in L^{2}(0,1)$, every time $T>0$, there exists a control $u \in C^{0}([0, T])$ such that the solution of 11.1$)$ satisfies $y(T, \cdot)=0$. By duality there exists $C_{T}>0$ (depending only on $T$ ) such that for every $z^{1} \in L^{2}(0,1)$, the solution $z$ of

$$
\begin{aligned}
-\dot{z}(t, x) & =\partial_{x}\left(p(x) \partial_{x} z(t, x)\right) z+q(x) z(t, x) & & (t \in(0, T), x \in(0,1)), \\
0 & =\alpha_{0} z(t, 0)+\alpha_{1} \partial_{x} z(t, 0) & & (t \in(0, T)), \\
0 & =\beta_{0} z(t, 1)+\beta_{1} \partial_{x} z(t, 1) & & (t \in(0, T)), \\
z(T, x) & =z^{1} & & (x \in(0,1)),
\end{aligned}
$$

satisfies,

if $\beta_{1} \neq 0$, or

$$
\|z(0, \cdot)\|_{L^{2}(0,1)} \leqslant C_{T}\|z(t, 1)\|_{L^{1}(0, T)},
$$

$$
\|z(0, \cdot)\|_{L^{2}(0,1)} \leqslant C_{T}\left\|\partial_{x} z(t, 1)\right\|_{L^{1}(0, T)},
$$

if $\beta_{0} \neq 0$. By duality, this means that the control $u$ steering $y^{0}$ to 0 in time $T$ can be chosen so that $\|u\|_{L^{\infty}(0, T)} \leqslant C_{T}\left\|y^{0}\right\|_{L^{2}(0,1)}$. It is then trivial exercise to see that for every $y^{1} \in \mathcal{S}_{+}^{*}$, the control $u$ steering $y^{0} \in L^{2}(0,1)$ to $y^{1}$ can be chosen so that

$$
\left\|u-u^{1}\right\|_{L^{\infty}(0, T)} \leqslant C_{T}\left\|y^{0}-y^{1}\right\|_{L^{2}(0,1)},
$$

where $u^{1}>0$ is the control associated to the steady state $y^{1}$.

When $L$ is dissipative. When $L$ is dissipative, it is a classical exercise to show that for every $\tau>0$ and every $k \in \mathbb{N}^{*}$, we have $C_{k \tau} \leqslant \frac{C_{\tau}}{k}$. Meaning in particular, for every $y^{1} \in \mathcal{S}_{+}^{*}$ and every $y^{0} \in L^{2}(0,1)$, that taking $\tau>0$ and taking $k \in \mathbb{N}^{*}$ sufficiently large, we have

$$
\left\|u-u^{1}\right\|_{L^{\infty}(0, T)} \leqslant \frac{C_{\tau}}{k}\left\|y^{0}-y^{1}\right\|_{L^{2}(0,1)} \leqslant u^{1}
$$

(here again $u^{1} \in \mathbb{R}_{+}^{*}$ is the control associated to the steady state $y^{1}$ ).

This in particular means that, by taking $k=k\left(y^{0}, y^{1}, \tau\right)$ sufficiently large, there exists a nonnegative control steering $y^{0}$ to $y^{1}$ in time $k \tau$. 
General situation. When $L$ is not dissipative, it is not possible to use the previous argument. However, we can use a quasi-static approach combined with a compactness argument. Indeed, given $\tau>0, y^{0}, y^{1} \in \mathcal{S}_{+}^{*}$, with associated control $u^{0}$ and $u^{1}$, for some $K \in \mathbb{N}^{*}$, we define, for every $k \in\{1, \ldots, K-1\}, y^{k / K} \in \mathcal{S}_{+}^{*}$ the steady state associated to the control $u^{0}+\frac{k}{K} u^{1}$. By taking $K$ large enough such that $\frac{C_{\tau}}{K}\left\|y^{1}-y^{0}\right\|_{L^{2}(0,1)} \leqslant \min \left\{u^{0}, u^{1}\right\}$, there exists a nonnegative control $u_{k}$ steering $y^{k / K}$ to $y^{(k+1) / K}$ in time $\tau$. Then by concatenating these controls, we have found a nonnegative control steering $y^{0}$ to $y^{1}$ in time $K \tau$.

\section{B Existence of a nonnegative minimal time control in the space of Radon measures}

The proof of this fact follows the one of [11, Theorems 2.1 and 3.1] or [12, Proposition 5.1.7]. Indeed, if $y^{0}$ and $y^{1} \in L^{2}(0,1)$ are such that $\underline{T}\left(y^{0}, y^{1}\right)<\infty$ (i.e., $y^{1}$ is reachable form $y^{0}$ with nonnegative controls), then there exists a nonincreasing sequence $\left(T_{k}\right)_{k \in \mathbb{N}} \in\left[\underline{T}\left(y^{0}, y^{1}\right), \infty\right)^{\mathbb{N}}$ such that $T_{k} \rightarrow$ $\underline{T}\left(y^{0}, y^{1}\right)$ as $k \rightarrow \infty$ and such hat for every $k \in \mathbb{N}$, there exists a nonnegative control $u_{k} \in L^{2}\left(0, T_{k}\right)$ steering the solution $y_{k}$ of $(1.1)$ from $y^{0}$ to $y^{1}$ in time $T_{k}$. In addition, since the operator $L$ defined by 1.3 is symmetric and diagonalizable, we pick an eigenvalue $\lambda \in \mathbb{R}$ of $L$ and $\varphi \in \mathcal{D}(L) \backslash\{0\}$ an associated eigenfunction. Let us define for every $i \in\{0,1\}, Y^{i}=\left\langle y^{i}, \varphi\right\rangle_{L^{2}(0,1), L^{2}(0,1)}$. Since $u_{k}$ is a control steering $y^{0}$ to $y^{1}$ in time $T_{k}$, we deduce that, $u_{k}$ shall satisfy $(2.2)$, with $y(T, \cdot)=y^{1}$, i.e.,

$$
\frac{Y^{1}-e^{\lambda T_{n}} Y^{0}}{\gamma}=\int_{0}^{T_{k}} e^{\lambda\left(T_{k}-t\right)} u_{k}(t) \mathrm{d} t \quad(k \in \mathbb{N}),
$$

with $\gamma=\left\{\begin{array}{ll}\frac{p(1)}{\beta_{1}} \varphi(1), & \text { if } \beta_{1} \neq 0, \\ -\frac{p(1)}{\beta_{0}} \partial_{x} \varphi(1), & \text { if } \beta_{0} \neq 0\end{array}\right.$ (recall that in both situations, we have $\left.\gamma \neq 0\right)$.

This ensures that (since $u_{k} \geqslant 0$ and $e^{\lambda\left(T_{k}-t\right)} \geqslant e^{-|\lambda| T_{k}}$ for every $t \in\left[0, T_{k}\right]$ ),

$$
\int_{0}^{T_{k}} u_{k}(t) \mathrm{d} t \leqslant e^{|\lambda| T_{k}} \frac{\left|Y^{1}\right|+e^{|\lambda| T_{k}}\left|Y^{0}\right|}{|\gamma|} .
$$

Finally, since $\left(T_{k}\right)_{k}$ is a nonincreasing sequence, we obtain (by extending $u_{k}$ by 0 on $\left(T_{k}, T_{0}\right)$ ) that,

$$
\left\|u_{k}\right\|_{L^{1}\left(0, T_{0}\right)} \leqslant e^{|\lambda| T_{0}} \frac{e^{|\lambda| T_{0}}\left|Y^{0}\right|+\left|Y^{1}\right|}{|\gamma|} \quad(k \in \mathbb{N}),
$$

that is to say that the sequence $\left(u_{k}\right)_{k}$ is uniformly bounded in $L^{1}\left(0, T_{0}\right)$ and hence, is up to the extraction of a subsequence, vaguely convergent to some Radon measure $\underline{u} \in \mathcal{M}\left(\left[0, T_{0}\right]\right)$ (see e.g., 3. Corollary 31.3 p. 206]). Since $u_{k} \geqslant 0$ and $\operatorname{supp} u_{k} \subset\left[0, T_{k}\right]$ for every $k \in \mathbb{N}$, we easily deduce that we necessarily have $\underline{u} \geqslant 0$ and $\operatorname{supp} \underline{u} \subset\left[0, \underline{T}\left(y^{0}, y^{1}\right)\right]$.

It remains to check that $\underline{u}$ is indeed a control steering $y^{0}$ to $y^{1}$ in time $\underline{T}\left(y^{0}, y^{1}\right)$. This can be deduced by taking the limit $\bar{k} \rightarrow \infty$ in (2.1) or 2.2 (with $T=T_{k}$ and $\left.\mathrm{d} \underline{u}(t)=u_{k}(t) \mathrm{d} t\right)$. 


\section{No gap situation}

Let $y^{0} \in L^{2}(0,1)$ and $y^{1} \in \mathcal{S}_{+}^{*}$ and assume that $\underline{T}\left(y^{0}, y^{1}\right)<\infty$, i.e. $y^{0}$ can be steered to $y^{1}$ with a nonnegative $L^{2}$ control. Recall that $\underline{T}\left(y^{0}, y^{1}\right)$ is defined by (1.4), i.e.,

$$
\begin{aligned}
\underline{T}\left(y^{0}, y^{1}\right)=\inf \left\{T>0 \mid \exists u \in L^{2}(0, T) \text { s.t. } u\right. & \geqslant 0 \\
& \text { and the solution } \left.y \text { of } 1.1 \text { satisfies } y(T, \cdot)=y^{1}\right\},
\end{aligned}
$$

The aim of this paragraph is to show that we have $\underline{T}\left(y^{0}, y^{1}=\underline{T}_{\mathcal{M}}\left(y^{0}, y^{1}\right)\right.$, where we have set

$$
\begin{array}{r}
\underline{T}_{\mathcal{M}}\left(y^{0}, y^{1}\right)=\inf \{T>0 \mid \exists \underline{u} \in \mathcal{M}([0, T]) \text { s.t. } \underline{u} \geqslant 0 \\
\left.\quad \text { and the solution } y \text { of } 1.1 \text { satisfies } y(T, \cdot)=y^{1}\right\},
\end{array}
$$

Obviously, we always have $0 \leqslant \underline{T}_{\mathcal{M}}\left(y^{0}, y^{1}\right) \leqslant \underline{T}\left(y^{0}, y^{1}\right)$. Arguing as in [12, Proposition 5.1.11] and using comments contained in Appendix $\mathrm{A}$, we can prove that, given a time $T \geqslant 0$ and a nonnegative control $\underline{u} \in \mathcal{M}([0, T])$ steering $y^{0}$ to $y^{1}$ in time $T$, and given any $\varepsilon>0$, there exists a control $u \in L^{2}(0, T+\varepsilon)$ steering $y^{0}$ to $y^{1}$ in time $T+\varepsilon$. This fact shows that $\underline{T}\left(y^{0}, y^{1}\right)=\underline{T}_{\mathcal{M}}\left(y^{0}, y^{1}\right)$.

Remark C.1. When $y^{1}$ is not a positive steady state, it has been shown (on some finite dimensional systems) in [12] that an infimum gap could occur (i.e., $\left.\underline{T}\left(y^{0}, y^{1}\right)<\underline{T}_{\mathcal{M}}\left(y^{0}, y^{1}\right)\right)$. Let us refer to 13 ] for general no gap condition for finite dimensional control systems.

\section{References}

[1] M. A. Al-Gwaiz. Sturm-Liouville theory and its applications. Springer Undergraduate Mathematics Series. Springer-Verlag London, Ltd., London, 2008.

[2] H. Antil, U. Biccari, R. Ponce, M. Warma, and S. Zamorano. Controllability properties from the exterior under positivity constraints for a 1-d fractional heat equation, 2019.

[3] H. Bauer. Measure and integration theory, volume 26 of De Gruyter Studies in Mathematics. Walter de Gruyter \& Co., Berlin, 2001. Translated from the German by Robert B. Burckel.

[4] U. Biccari, M. Warma, and E. Zuazua. Controllability of the one-dimensional fractional heat equation under positivity constraints, 2019.

[5] E. Casas. Pontryagin's principle for state-constrained boundary control problems of semilinear parabolic equations. SIAM J. Control Optim., 35(4):1297-1327, 1997.

[6] N. Dunford and J. T. Schwartz. Linear operators. Part II. Wiley Classics Library. John Wiley \& Sons, Inc., New York, 1988. Spectral theory. Selfadjoint operators in Hilbert space, With the assistance of William G. Bade and Robert G. Bartle, Reprint of the 1963 original, A Wiley-Interscience Publication.

[7] H. Fattorini and D. Russell. Exact controllability theorems for linear parabolic equations in one space dimension. Arch. Ration. Mech. Anal., 43:272-292, 1971.

[8] E. Fernández-Cara, M. González-Burgos, and L. de Teresa. Boundary controllability of parabolic coupled equations. J. Funct. Anal., 259(7):1720-1758, 2010. 
[9] E. Lee and L. Markus. Foundations of optimal control theory. (The SIAM Series in Applied Mathematics.) New York-London-Sydney: John Wiley and Sons, Inc. XII, 576 p. (1967)., 1967.

[10] P. Lissy and C. Moreau. State-constrained controllability of linear reaction-diffusion systems. working paper or preprint, Nov. 2020.

[11] J. Lohéac, E. Trélat, and E. Zuazua. Minimal controllability time for the heat equation under unilateral state or control constraints. Mathematical Models and Methods in Applied Sciences, 27(09):1587-1644, 2017.

[12] J. Lohéac, E. Trélat, and E. Zuazua. Nonnegative control of finite-dimensional linear systems. working paper or preprint, 2019.

[13] M. Motta, F. Rampazzo, and R. Vinter. Normality and gap phenomena in optimal unbounded control. ESAIM Control Optim. Calc. Var., 24(4):1645-1673, 2018.

[14] D. Pighin and E. Zuazua. Controllability under positivity constraints of semilinear heat equations. Math. Control Relat. Fields, 8(3-4):935-964, 2018.

[15] D. Pighin and E. Zuazua. Controllability under positivity constraints of multi-d wave equations. In Trends in control theory and partial differential equations, volume 32 of Springer INdAM Ser., pages 195-232. Springer, Cham, 2019.

[16] E. Schmidt. Boundary control for the heat equation with steady-state targets. SIAM J. Control Optim., 18:145-154, 1980.

[17] M. Tucsnak and G. Weiss. Observation and control for operator semigroups. Basel: Birkhäuser, 2009. 